\title{
A Family Tree of Optical Transients from Narrow-line Seyfert 1 Galaxies
}

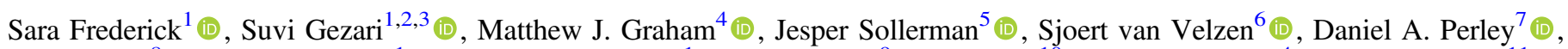

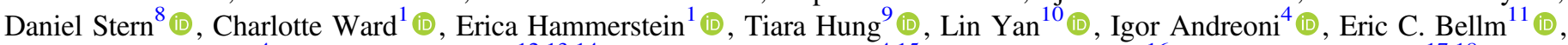 \\ Dmitry A. Duev ${ }^{4}$ (D), Marek Kowalski ${ }^{12,13,14}$ (D), Ashish A. Mahabal ${ }^{4,15}$ (D), Frank J. Masci ${ }^{16}$ (D), Michael Medford ${ }^{17,18}$ (D), \\ Ben Rusholme ${ }^{16}$ (D) , Roger Smith ${ }^{10}\left(\mathbb{D}\right.$, and Richard Walters ${ }^{10}$ \\ ${ }^{1}$ Department of Astronomy, University of Maryland, College Park, MD 20742, USA; sfrederick@astro.umd.edu \\ 2 Joint Space-Science Institute, University of Maryland, College Park, MD 20742, USA \\ ${ }^{3}$ Space Telescope Science Institute, Baltimore, MD 21218, USA \\ ${ }^{4}$ Division of Physics, Mathematics and Astronomy, California Institute of Technology, Pasadena, CA 91125, USA \\ ${ }^{5}$ The Oskar Klein Centre \& Department of Astronomy, Stockholm University, AlbaNova, SE-106 91 Stockholm, Sweden \\ ${ }^{6}$ Leiden Observatory, Leiden University, P.O. Box 9513, 2300 RA Leiden, The Netherlands \\ ${ }^{7}$ Astrophysics Research Institute, Liverpool John Moores University, 146 Brownlow Hill, Liverpool L3 5RF, UK \\ 8 Jet Propulsion Laboratory, California Institute of Technology, 4800 Oak Grove Drive, Mail Stop 169-221, Pasadena, CA 91109, USA \\ ${ }^{9}$ Department of Astronomy and Astrophysics, University of California, Santa Cruz, CA 95064, USA \\ ${ }^{10}$ Caltech Optical Observatories, California Institute of Technology, Pasadena, CA 91125, USA \\ ${ }^{11}$ DIRAC Institute, Department of Astronomy, University of Washington, 3910 15th Avenue NE, Seattle, WA 98195, USA \\ ${ }_{12}$ Deutsches Elektronen Synchrotron DESY, Platanenallee 6, 15738 Zeuthen, Germany \\ ${ }^{13}$ Institut für Physik, Humboldt-Universität zu Berlin, D-12489 Berlin, Germany \\ ${ }^{14}$ Columbia Astrophysics Laboratory, Columbia University in the City of New York, 550 W 120th St., New York, NY 10027, USA \\ ${ }_{15}^{15}$ Center for Data Driven Discovery, California Institute of Technology, Pasadena, CA 91125, USA \\ ${ }^{16}$ IPAC, California Institute of Technology, 1200 E. California Boulevard, Pasadena, CA 91125, USA \\ ${ }^{17}$ University of California, Berkeley, Department of Astronomy, Berkeley, CA 94720, USA \\ ${ }^{18}$ Lawrence Berkeley National Laboratory, 1 Cyclotron Road, Berkeley, CA 94720, USA \\ Received 2020 October 16; revised 2021 June 30; accepted 2021 July 1; published 2021 October 12
}

\begin{abstract}
The Zwicky Transient Facility (ZTF) has discovered five events $(0.01<z<0.4)$ belonging to an emerging class of active galactic nuclei (AGNs) undergoing smooth, large-amplitude, and rapidly rising flares. This sample consists of several transients initially classified as supernovae with narrow spectral lines. However, upon closer inspection, all of the host galaxies display Balmer lines with $\mathrm{FWHM}(\mathrm{H} \beta) \sim 900-1400 \mathrm{~km} \mathrm{~s}^{-1}$, characteristic of a narrow-line Seyfert 1 (NLSy1) galaxy. The transient events are long lived, over 400 days on average in the observed frame. We report UV and X-ray follow-up of the flares and observe persistent UV emission, with two of the five transients detected with luminous X-ray emission, ruling out a supernova interpretation. We compare the properties of this sample to previously reported flaring NLSy1 galaxies and find that they fall into three spectroscopic categories: 1) Balmer line profiles and Fe II complexes typical of NLSy1s, 2) strong He II profiles, and 3) He II profiles including Bowen fluorescence features. The latter are members of the growing class of AGN flares attributed to enhanced accretion reported by Trakhtenbrot et al. We consider physical interpretations in the context of related transients from the literature. For example, two of the sources show high-amplitude rebrightening in the optical, ruling out a simple tidal disruption event scenario for those transients. We conclude that three of the sample belong to the Trakhtenbrot et al. class and two are tidal disruption events in NLSy1s. We also hypothesize as to why NLSy1s are preferentially the sites of such rapid enhanced flaring activity.
\end{abstract}

Unified Astronomy Thesaurus concepts: Accretion (14); Active galaxies (17); AGN host galaxies (2017); Active galactic nuclei (16); Seyfert galaxies (1447); High energy astrophysics (739)

\section{Introduction}

A galaxy center hosting an unobscured, or Type 1, active galactic nucleus (AGN) is dominated by its continuum emission. Therefore, a flare originating from this nuclear region requires a distinctly powerful event to be detectable above this stochastically variable continuum. A small number of rapid, ${ }^{19}$ smoothly evolving flares have been observed to be associated with AGNs (e.g., Drake et al. 2011; Blanchard et al. 2017), with few known mechanisms that can cause these events to occur.

Intrinsic UV/optical flares, such as those due to enhanced accretion onto the central supermassive black hole (SMBH) in the form of gaseous material or stars passing too close to the

\footnotetext{
19 We refer to flare timescales as "rapid" when they occur on a week to month timescales.
}

nucleus, have been observed in the form of tidal disruption events (TDEs; see the recent review by van Velzen et al. 2021 and references therein), UV-bright flaring events that are associated with accretion rate changes (Trakhtenbrot et al. 2019a), transients with double-peaked line profiles linked to accretion disk emission (e.g., Halpern \& Eracleous 1994), or changing-look AGNs-the dramatic change in spectroscopic AGN classification following a rise in continuum level, thought to be connected to unstable changes in accretion state (e.g., LaMassa et al. 2015; MacLeod et al. 2016; Ruan et al. 2016; Runnoe et al. 2016; Ross et al. 2018; Stern et al. 2018; Frederick et al. 2019; Trakhtenbrot et al. 2019b; Graham et al. 2020 and references therein). There also exists the intriguing possibility of links between these classes, such as TDEs occurring in preexisting AGN disks (e.g., Merloni et al. 2015; Chan et al. 2019, 2020). 
Phenomena extrinsic to the SMBH accretion engine, such as microlensing of a quasar by a foreground Galactic source (e.g., Lawrence et al. 2012) or slowly evolving superluminous supernova (SLSN) explosions, have also been observed to cause smooth large-amplitude flares from galaxies with AGNs (Graham et al. 2017). In rare cases these can be astrometrically indistinguishable from the galactic nucleus, and therefore, it becomes difficult to discern whether an explosive disruption to the accretion flow has occurred and to differentiate this from AGN variability (Terlevich et al. 1992).

Multiwavelength approaches are required to disentangle this diverse family of observed flaring behaviors from AGNs. In the golden era of time domain astronomy, even with many multichromatic instruments trained on the sky, a number of newly discovered objects continue to defy placement into a clear-cut observational category.

In order of discovery, we present a photometric class composed of five rapid flares with similar smooth light-curve shapes occurring in a subclass of AGNs observed by the Zwicky Transient Facility (ZTF) survey:

(a) ZTF19aailpwl/AT2019brs $(z=0.37362)$

(b) ZTF19abvgxrq/AT2019pev $(z=0.097)$

(c) ZTF19aatubsj/AT2019fdr $(z=0.2666)$

(d) ZTF19aaiqmgl/AT2019avd $(z=0.0296)$

(e) ZTF18abjjkeo/AT2020hle $(z=0.103)$

In Section 2 we present the follow-up of these flares. In Section 3 we compare the results of their respective multiwavelength follow-up campaigns to observations of a variety of related objects found in recent years, and in Section 4 we attempt to place them into a classification scheme based on observational properties, summarized in Section 5. All transients in the sample are referred to by their International Astronomical Union (IAU) recognized transient designations throughout. All magnitudes are reported in the AB system and light curves are shown in the observed frame unless otherwise stated. We have adopted the following cosmology: $H_{0}=$ $70 \mathrm{~km} \mathrm{~s}^{-1} \mathrm{Mpc}^{-1}, \Omega_{\Lambda}=0.73$, and $\Omega_{M}=0.27$.

\section{Observations}

The ZTF Survey (Graham et al. 2019; Bellm et al. 2019a) comprises the automated Palomar 48 inch Samuel Oschin Telescope (P48) as well as the Palomar 60 inch Spectral Energy Distribution Machine (P60 SEDM; Blagorodnova et al. 2018; Rigault et al. 2019) integral field unit spectrograph and has surveyed the Northern Sky with $g$ - and $r$-band filters with a three-night cadence since 2018 (Bellm et al. 2019b). At least 15 images meeting good-quality criteria were stacked to build a coadded reference image of each observing field and quadrant in each filter band. Science images are subtracted by their references and processed each night by the Infrared Processing and Analysis Center (IPAC) pipeline (Masci et al. 2019). The candidate transient alert stream (Patterson et al. 2019) is distributed by the University of Washington Kafka system and filtered through the AGN and BH Science Working Group's Nuclear Transients ${ }^{20}$ parameter criteria (outlined in van Velzen et al. 2019, 2021) by the Ampel broker (Soumagnac \& Ofek 2018; Nordin et al. 2019), with the GROWTH Marshal

\footnotetext{
${ }^{20}$ A nuclear transient was defined as that within $0 . / 5$ of the reference galaxy center. Over 9000 nuclear transients passed this filter and were ranked during ZTF Phase I, of which 27 were TDEs, over $7 \%$ were classified as SN, and over half were AGN or candidate AGN.
}

user interface utilized for the coordination of follow-up efforts (Kasliwal et al. 2019).

All 5 transients included in the sample presented here were selected based on the following criteria: large-amplitude, nuclear variability $(\Delta g>1 \mathrm{mag}$ in difference imaging photometry, and within 0 ". 5 of the center of the host galaxy in the reference image) with follow-up or preflare spectra consistent with an AGN classification. This selection was not systematic (and therefore not complete), but rather the result of ongoing intersecting and collaborative searches for changing-look AGN (Frederick et al. 2019), TDEs (van Velzen et al. 2019, 2021), and superluminous supernovae (SLSNe; Lunnan et al. 2020; Yan et al. 2020) relying on partial human vetting from the ZTF transient alert stream, from which this sample emerged as more examples were collected. A systematic search for NLSy1 transients in ZTF will be the focus of a future study.

\subsection{Optical Photometry}

All transients in the sample were detected prepeak using ZTF difference imaging photometry. The smooth light-curve shapes (with scatter $\Delta g<0.1 \mathrm{mag}$ ) of the sample are shown in Figure 1. All magnitude changes are reported in the $g$ band unless otherwise noted. An analysis of the rise times to peak are measured and reported in Section 3.1.1. We report the $g$-band magnitude-weighted offsets for each transient, calculated using Equation (3) in van Velzen et al. (2019). ZTF forced photometry for the sample is shown in Figure 11 of the Appendix.

AT2019brs-(R.A. $=14: 27: 46.41, \quad$ decl $=+29: 30: 38.6$, J2000.0), also known as ZTF19aailpwl, it was first detected on 2019 February 08 as a nuclear transient within 0 ". 17 of the host galaxy center.

AT2019pev-(R.A. $=04: 29: 22.72, \quad$ decl $=+00: 37: 07.6$, J2000.0) also known as ZTF19abvgxrq and Gaia19eby, it was first detected on 2019 September 4 as a nuclear transient within 0 ." 15 of the host galaxy center. ATLAS, Gaia, and PanSTARRs also reported observations of this source on the Transient Name Server (TNS) with discovery dates of 2019 September 4, 2019 September 13, and 2019 September 26, respectively. The host galaxy displayed no variability above the 0.5 mag level in CRTS.

$A T 2019 f d r-$ (R.A. $=17: 09: 06.86, \quad$ decl. $=+26: 51: 20.7$, J2000.0) also known as ZTF19aatubsj, it was detected on 2019 April 27 with a significant flux increase with respect to the reference image and with an offset from the nucleus of its host of 0 ". 13 . During a coverage gap in the first 40 days of the rise, ATLAS reported an intriguing "bump" feature (Smartt et al. 2019). The host galaxy displayed variability at the 2 mag level in $V$-band CRTS data from 2009 to 2013 (variability which was not observed in ZTF forced photometry prior to the transient).

AT2019avd-(R.A. $=08: 23: 36.77, \quad$ decl. $=+04: 23: 02.5$, J2000.0) also known as eRASSt J082337+042303 ${ }^{21}$ and ZTF19aaiqmgl, it was detected by ZTF beginning on 2019 February 9 within 0 ." 06 of its host galaxy. The host showed no variability in CRTS for $15 \mathrm{yr}$ prior to its rapid rise to peak.

\footnotetext{
21 This was the only source in the sample to be detected by the extended ROentgen Survey with an Imaging Telescope Array (eROSITA, part of the Russian-German "Spectrum-Roentgen-Gamma" (SRG) mission; Predehl et al. 2021) and was given the name eRASSt J082337+042303. This X-ray detection coincident with the transient's host galaxy is described in Section 2.4.
} 


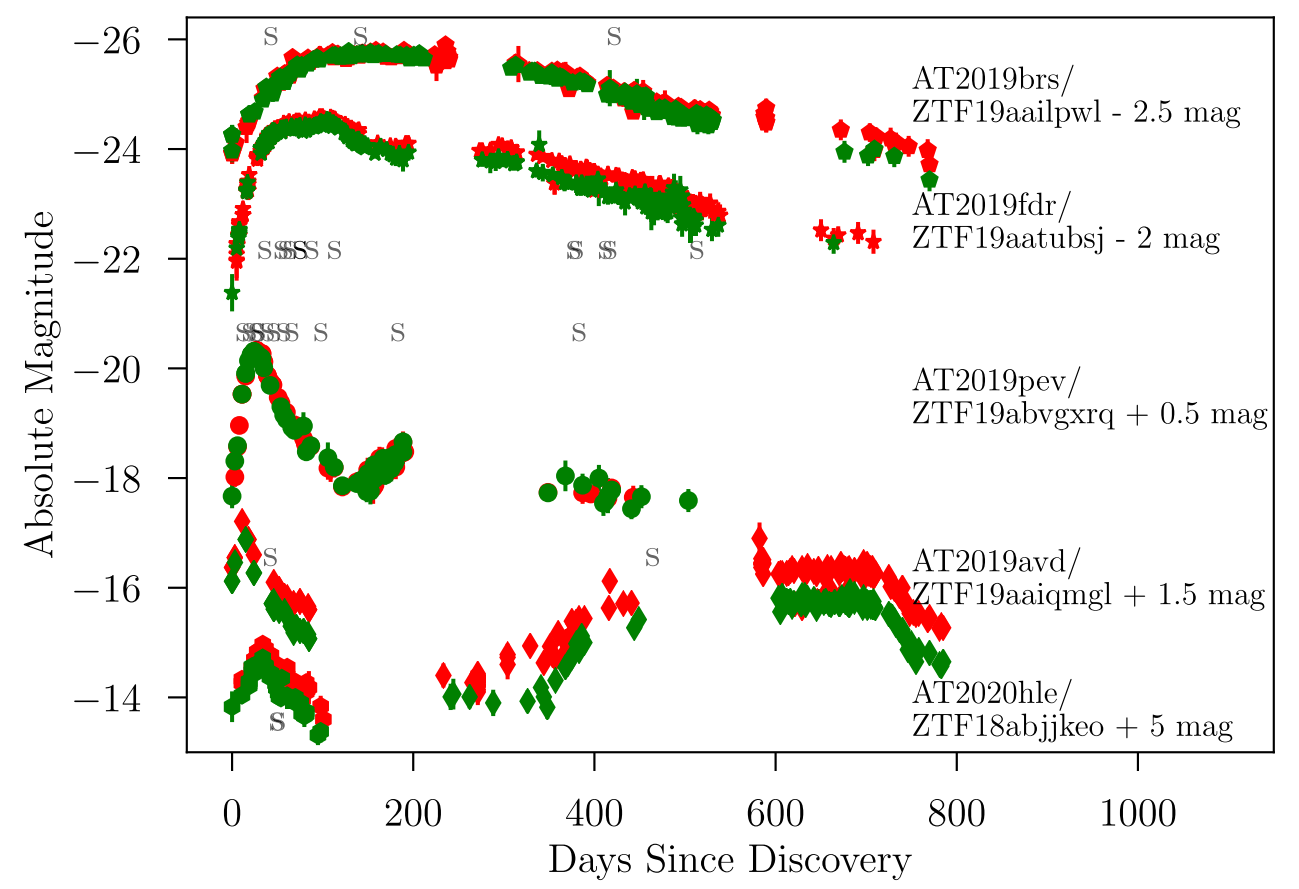

Figure 1. Comparison of the ZTF $g$ - and $r$-band difference imaging light-curve shapes and absolute magnitudes of the sample. AT2019fdr decreases before reaching a second plateau stage and undergoes significant reddening after the first plateau while the others never do. AT2019pev rises again symmetrically after decreasing to preflare levels, as does AT2019avd. The light curves have been shifted in absolute magnitude space for visual purposes, as indicated alongside the object name. Overlap of the $g$ and $r$ light curves reflects true colors such that the initial colors approach $g-r=0$ mag for all transients in the sample. Observations at other wavelengths are shown in Figure 2. Spectroscopic epochs are labeled for each light curve with an "S" below AT2019fdr and AT2020hle and above the rest.

AT2020hle-(R.A. $=11: 07: 42.91, \quad$ decl. $=+74: 38: 02.0$, J2000.0) also known as ZTF18abjjkeo, it was detected beginning on 2020 April 05 within 0 " 02 of its host galaxy center. The ZTF forced photometry for this source shows no variability above the level of the galaxy for $>400$ days. The host galaxy of AT2020hle was beyond the survey limits of CRTS.

\subsection{Optical Spectroscopy}

All spectroscopic follow-up observations for the sample are summarized in Table 1, and each epoch is shown in Figure 12 of the Appendix. The spectroscopic data of the sample shown in Figures 7 and 6 are archived online at the Weizmann Interactive Supernova data REPository (WISeREP; Yaron \& Gal-Yam 2012). ${ }^{22}$ The phases of the optical follow-up spectra with respect to the features in the ZTF light curves are annotated in Figure 1. All transients in this sample have spectral characteristics of NLSy1 galaxies, i.e., strong Balmer line emission with FWHM $<2000 \mathrm{~km} \mathrm{~s}^{-1}$, along with other spectral features that are highlighted below and explored in detail in Section 3.2.

Spectra taken with the Lowell Discovery Telescope (LDT; formerly DCT) Deveny spectrograph (PI: Gezari), the Alhambra Faint Object Spectrograph and Camera (ALFOSC) on the $2.56 \mathrm{~m}$ Nordic Optical Telescope (NOT; PI: Sollerman), and the KAST Double Spectrograph on the Lick $3 \mathrm{~m}$ Shane Telescope (PI: Foley) were reduced using standard IRAF procedures including wavelength calibration using arc lamps and flux calibration using a standard star. Spectra obtained with the Low-Resolution Imaging Spectrometer (LRIS) on the Keck I $10 \mathrm{~m}$ telescope were reduced automatically with the LRIS reduction pipeline Lpipe Perley (2019). Spectroscopy

\footnotetext{
22 https://www.wiserep.org/
}

obtained with the Double Beam Spectrograph (DBSP) on the Palomar 200 inch Hale Telescope (P200; PI: Yan) were reduced using the pyraf-dbsp pipeline (Science Software Branch at STScI 2012; Bellm \& Sesar 2016). Folded Low Order whYte-pupil Double-dispersed Spectrograph North (FLOYDS-N) spectra were obtained from the TNS (Arcavi et al. 2019). Data taken by the robotic $2 \mathrm{~m}$ Liverpool Telescope (LT) SPectrograph for the Rapid Acquisition of Transients (SPRAT; PI: Perley) were reduced by the standard pipeline provided by the Observatorio del Roque de los Muchachos. Lower-resolution $(R \sim 100)$ spectra taken by the Palomar 60 inch SEDM (Program PIs: Gezari, Sollerman, Kulkarni) were reduced automatically with pysedm (Rigault et al. 2019).

AT2019brs - showed dramatic changes relative to the 2006 SDSS spectrum, which had already identified the host galaxy as an NLSy1 (Rakshit et al. 2017; Abolfathi et al. 2018).

The follow-up FLOYDS-N (Arcavi et al. 2019) and LDT (PI: Gezari) spectra showed a steep blue continuum and a strong H II profile with Bowen fluorescence features, indicating it became a flaring SMBH belonging to the observational class established by Trakhtenbrot et al. (2019a).

AT2019pev-spectroscopically identified as an NLSy1 on 2019 September 15 with LT SPRAT (PI: Perley), based on the width of the Balmer emission lines and the strength of the [O III] $\lambda 5007$ emission line. Gezari et al. (2019) reported that the LT spectrum showed evidence for blueshifted H II $\lambda 4686$ emission as well as N III $\lambda 4640$ emission, due to the Bowen fluorescence mechanism, placing it again in the observational subclass of the Trakhtenbrot et al. (2019a) objects. Near peak it was observed with the Keck 10 m Low Resolution Imaging Spectrometer (LRIS; PI: Graham) as well as the LDT Deveny Spectrograph (PI: Gezari) and Lick (PI: Foley), which confirmed the strong blue continuum and clearly defined and persistent Bowen fluorescence features. 
Table 1

Summary of Spectroscopic Follow-up Observations of the Sample

\begin{tabular}{|c|c|c|c|c|}
\hline Name & Obs UT & Instrument & Exposure (s) & Reference \\
\hline AT2019brs & $\begin{array}{l}2006 \text { Jul } 01 \\
2019 \text { Mar } 15 \\
\text { 2019 Jun } 22 \\
\text { 2020 Mar 28 } \\
\text { 2020 Dec 06 }\end{array}$ & $\begin{array}{c}\text { SDSS } \\
\text { FLOYDS-N } \\
\text { LDT Deveny } \\
\text { Palomar } 60^{\prime \prime} \text { SEDM } \\
\text { LDT Deveny }\end{array}$ & $\begin{array}{c}3000 \\
3600 \\
900 \\
2250 \\
900\end{array}$ & $\begin{array}{l}\text { Abolfathi et al. (2018) } \\
\text { Arcavi et al. (2019) } \\
\text { This work } \\
\text { This work } \\
\text { This work }\end{array}$ \\
\hline AT2019pev & $\begin{array}{l}\text { 2019 Sep 08 } \\
\text { 2019 Sep } 15 \\
\text { 2019 Sep } 22 \\
\text { 2019 Sep } 24 \\
\text { 2019 Sep } 25 \\
\text { 2019 Sep } 25 \\
2019 \text { Oct } 01 \\
2019 \text { Oct } 05 \\
2019 \text { Oct } 12 \\
\text { 2019 Oct } 15 \\
\text { 2019 Oct } 23 \\
\text { 2019 Nov 01 } \\
\text { 2019 Dec 03 } \\
\text { 2020 Jan } 30 \\
\text { 2020 Feb 26 } \\
\text { 2020 Sep } 13\end{array}$ & $\begin{array}{c}\text { Palomar 60" SEDM } \\
\text { LT SPRAT } \\
\text { Palomar 60" SEDM } \\
\text { LDT Deveny } \\
\text { Keck LRIS } \\
\text { NICER } \\
\text { Chandra LETG } \\
\text { Lick } 3 \text { m KAST } \\
\text { LT SPRAT } \\
\text { Chandra LETG } \\
\text { LDT Deveny } \\
\text { Palomar 60" SEDM } \\
\text { LDT Deveny } \\
\text { Swift XRT } \\
\text { LDT Deveny } \\
\text { LDT Deveny }\end{array}$ & $\begin{array}{c}2250 \\
500 \\
2250 \\
600 \\
300 \\
2000 \\
45400 \\
1500 \\
500 \\
91000 \\
900 \\
2250 \\
2400 \\
94700 \\
2600 \\
3200\end{array}$ & $\begin{array}{c}\text { This work } \\
\text { This work } \\
\text { This work } \\
\text { This work } \\
\text { This work } \\
\text { Kara et al. (2019) } \\
\text { Miller et al. (2019) } \\
\text { This work } \\
\text { This work } \\
\text { Mathur et al. (2019) } \\
\text { This work } \\
\text { This work } \\
\text { This work } \\
\text { This work } \\
\text { This work } \\
\text { This work }\end{array}$ \\
\hline AT2019fdr & $\begin{array}{l}\text { 2019 May } 25 \\
\text { 2019 Jun } 17 \\
\text { 2019 Jun } 22 \\
\text { 2019 Jul 03 } \\
\text { 2020 Apr } 30 \\
\text { 2020 Jun 04 } \\
\text { 2020 Jun 09 } \\
\text { 2020 Sep } 15\end{array}$ & $\begin{array}{c}\text { Palomar } 60^{\prime \prime} \text { SEDM } \\
\text { LT SPRAT } \\
\text { LDT Deveny } \\
\text { Palomar 200" Hale } \\
\text { NOT ALFOSC } \\
\text { Palomar 60" SEDM } \\
\text { LDT Deveny } \\
\text { LDT Deveny }\end{array}$ & $\begin{array}{c}2250 \\
900 \\
900 \\
600 \\
1750 \\
2250 \\
900 \\
900\end{array}$ & $\begin{array}{l}\text { This work } \\
\text { This work } \\
\text { This work } \\
\text { This work } \\
\text { This work } \\
\text { This work } \\
\text { This work } \\
\text { This work }\end{array}$ \\
\hline AT2019avd & $\begin{array}{l}2020 \text { Mar } 15 \\
2020 \text { Apr } 28 \\
\text { 2020 May } 10 \\
\text { 2019 Sep } 18 \\
\text { 2019 Sep } 19 \\
\text { 2019 Sep } 22\end{array}$ & $\begin{array}{c}\text { NOT ALFOSC } \\
\text { SRG eROSITA } \\
\text { FLOYDS-S } \\
\text { Palomar 60" SEDM } \\
\text { Palomar 60" SEDM } \\
\text { Palomar 60" SEDM }\end{array}$ & $\begin{array}{c}1800 \\
140 \\
3600 \\
2250 \\
2250 \\
2250\end{array}$ & $\begin{array}{l}\text { Malyali et al. (2021) } \\
\text { Malyali et al. (2021) } \\
\text { Trakhtenbrot et al. (2020) } \\
\text { This work } \\
\text { This work } \\
\text { This work }\end{array}$ \\
\hline AT2020hle & $\begin{array}{l}2020 \text { May } 16 \\
2020 \text { May } 18 \\
2020 \text { Dec } 06\end{array}$ & $\begin{array}{c}\text { Palomar } 60^{\prime \prime} \text { SEDM } \\
\text { LT SPRAT } \\
\text { LDT Deveny }\end{array}$ & $\begin{array}{l}2250 \\
1000 \\
2400\end{array}$ & $\begin{array}{l}\text { This work } \\
\text { This work } \\
\text { This work }\end{array}$ \\
\hline
\end{tabular}

AT2019fdr-observed 8 days after peak on 2019 July 03 with the P200 DBSP (PI: Yan). We measured a significant "blue horn" component of $\mathrm{H} \beta$ and marginally detected H II. The transient continuum of AT2019fdrfaded to reveal an underlying Fe II complex in the NOT ALFOSC (PI: Sollerman) spectrum taken nearly 368 days after peak on 2020 April 30, with no evidence for $\mathrm{H}$ II emission.

AT2019avd-The spectrum taken with NOT (PI: Sollerman) on 2019 March 15 near the first optical peak showed strong Balmer line emission, no detection of a $\mathrm{H}$ II line complex, and evidence for a Fe II complex, characteristic of NLSy1 galaxies. A follow-up FLOYDS-S spectrum taken 444 days after peak and reported to the TNS by Trakhtenbrot et al. (2020) showed the appearance of $\mathrm{H}$ II and Bowen fluorescence features and a "blue horn" in $\mathrm{H} \beta$. Again this event was classified as a member of the Trakhtenbrot et al. (2019a) observational class of flaring NLSy1s.

AT2020hle - In the LT (PI: Perley) spectrum of AT2020hle taken on 2020 May 18, 8 days after peak, the narrow component of the H II profile is significantly blueshifted. No Fe II line complex was detected in the spectra of this transient.

\subsection{UV Photometry}

We triggered target-of-opportunity monitoring observations with the Neil Gehrels Swift Telescope (Gehrels et al. 2004) for all transients in the sample. Using the HEASOFT command uvotsource, we extracted UVOT photometry within a $5^{\prime \prime}$ radius circular aperture and using an annular background region centered on the coordinates of the optical transient.

Figure 2 shows the $\nu L_{\nu}$ light curves of all flares in the sample. We compare ZTF $g$ - and $r$-band difference imaging, Wide-field Infrared Survey Explorer (WISE) difference imaging, Swift X-ray Telescope (XRT) monitoring, and Swift UVOT detections subtracted by the archival Galaxy Evolution Explorer (GALEX; Martin et al. 2005) All-Sky Imaging Survey (AIS; Bianchi et al. 2017) near-UV (NUV; $\lambda_{\text {eff }}=2310 \AA$ ) host measurements (measured with a $6 "$ radius aperture). The GALEX coadds include observations of the host galaxies taken throughout the survey duration between 2003 and 2013.

We found all transients in the sample to be UV detected, but with varying UV colors. The UV color of AT2019avd 

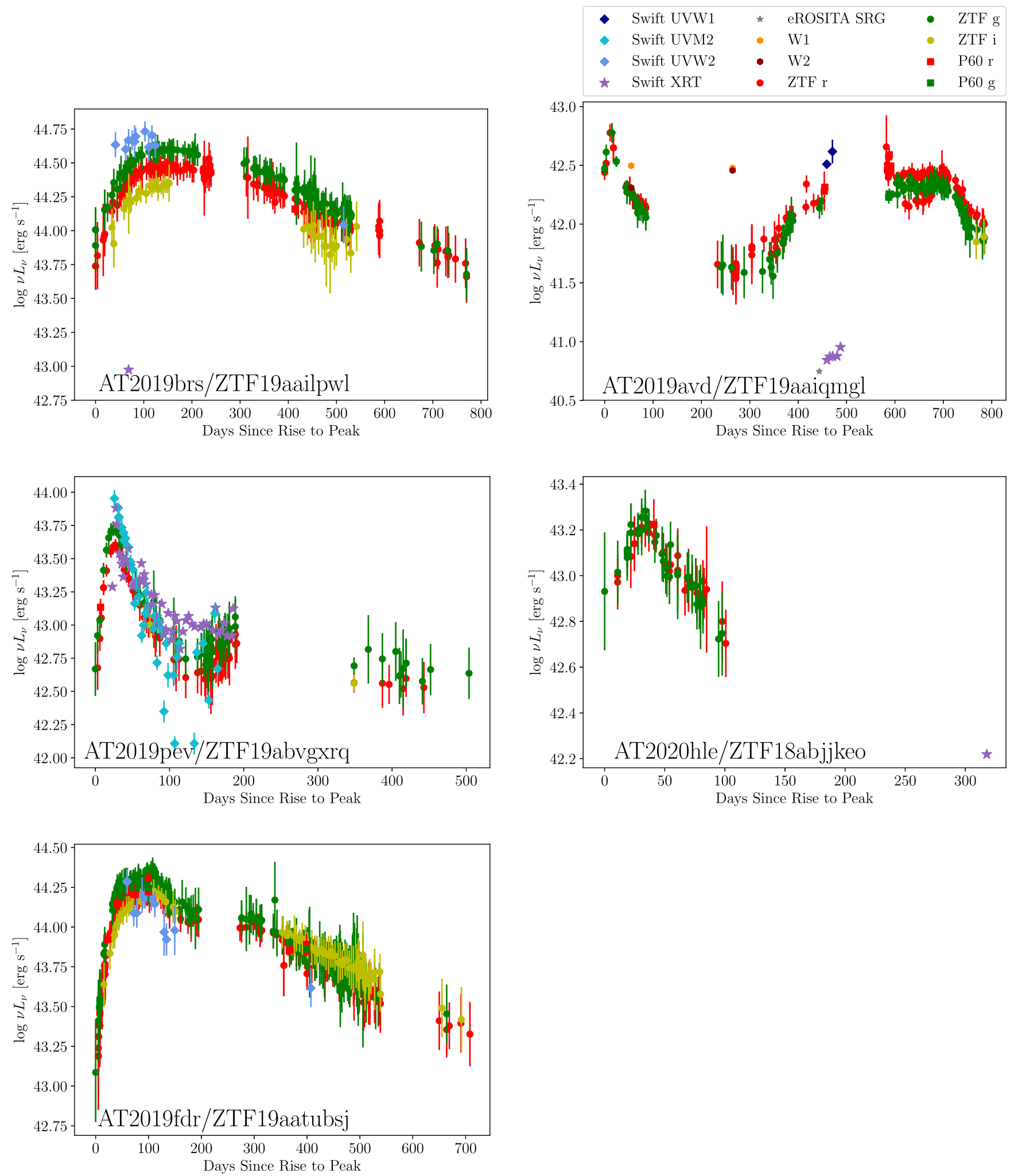

Figure 2. We track the colors of the transients in the sample with a $\nu L_{\nu}$ light curve, comparing the ZTF and WISE data to concurrent high-cadence Swift UVOT and XRT monitoring observations. The X-ray rise and fade of AT2019pev tracks the optical/UV with no significant delay. Times are given in days since the first ZTF detection, and data have been host/reference-subtracted. The X-ray error bars are comparable to the size of the data points. See Figure 11 for pre-outburst forced photometry.

(UVW1 $-g=-0.2 \mathrm{mag}$ ) was similar to that of AT2019pev (UVW2 $-g=-0.2 \mathrm{mag}$ ) and AT2019brs (ranging from UVW2 $-g=-0.1$ mag to $-0.7 \mathrm{mag}$ in 80 days) but
AT2019fdr was the only transient in the sample with red UV color (UVW2 $-g=0.8 \mathrm{mag}$ ). The UV light curves of the sample tend to follow the shape of the optical. 
AT2019pev became host dominated over time as the transient faded, but with strong scatter in the light curve as it approached the host magnitude.

\subsection{X-Rays}

We found only two transients in the sample to be detected in the X-rays in follow-up Swift XRT observations: AT2019pev and AT2019avd. AT2019brs was detected only once, and then only at a low level. We measured an XRT upper limit of 0.004 counts $\mathrm{s}^{-1}$ for AT2019fdr. X-ray follow-up spectra are reported in Table 1 . Swift photometry compared to WISE $W 1$ - and $W 2$-band and ZTF $g$ and $r$-band photometry is shown in Figure 2. The X-ray flares in this sample tend to vary in lockstep with the slow UV/optical flares. We compute unabsorbed X-ray flux densities at $2 \mathrm{keV}$ using the Portable Interactive Multi-Mission Simulator (PIMMS; Mukai 1993) $\mathrm{v} 4.10$ web tool ${ }^{23}$ and report X-ray-UV optical spectral index ratios in Section 3.1.3.

AT2019brs - detected only once in 11 observations during a 16 month monitoring campaign between 2019 March 21 and 2020 July 7 . We measured a $3 \sigma$ detection of 0.003 counts s $^{-1}$ (or $1.00 \times 10^{-13} \mathrm{erg} \mathrm{s}^{-1} \mathrm{~cm}^{-2}$ at $0.3-10 \mathrm{keV}$ ) on 18 April 2019, just brighter than the limiting flux. The XMM-Newton Slew Survey also reported an upper limit on 10 Jan 2021 of $<1.05 \times 10^{-11} \mathrm{erg} \mathrm{s}^{-1} \mathrm{~cm}^{-2}$ flux at $2-12 \mathrm{keV} .^{24}$

AT2019pev-Similar to the UV light curve, the shape of the X-ray flare of AT2019pev followed the optical, from its fade through its second rise 100 days after peak (See Figure 2 and Section 3). The unabsorbed $0.3-10 \mathrm{keV}$ flux from the stacked XRT spectrum of AT2019pev including data between 23 and 190 days after detection was $7.3 \pm 0.1 \times 10^{-12} \mathrm{erg} \mathrm{cm}^{-2} \mathrm{~s}^{-1}$. AT2019pev was previously detected in ROSAT (Voges et al. 1999), $29 \mathrm{yr}$ prior to the optical transient, and NICER observations taken within 1 day of the XRT detection show an increase in flux from this by 100 times $\left(1 \times 10^{-11} \mathrm{erg} \mathrm{cm}^{-2}\right.$ $\mathrm{s}^{-1}$ ), variable from 11 to 14 counts $\mathrm{s}^{-1}$ in $3 \mathrm{hr}$ (Kara et al. 2019). A 50 ks Chandra LETG grating observation taken just 8 days after peak and reported by Miller et al. (2019) found a flux consistent with this, with the spectral shape a good fit to a $k T=0.24 \mathrm{keV}$ blackbody, and the source variable at the $25 \%$ level on 2-3 ks timescales. Mathur et al. (2019) reported a decrease in $0.3-2.5 \mathrm{keV}$ flux to $7.7 \times 10^{-12} \mathrm{erg} \mathrm{cm}^{-2} \mathrm{~s}^{-1}$; their 91 ks Chandra LETG observation taken 42-43 days after the transient detection was a good fit to a consistent blackbody model and a power-law component typical of AGNs with spectral index $\Gamma=1.8$, with no intrinsic absorption required.

AT2019avd - observed only during the second optical flare (on 2020 April 28, 350 days after the first ZTF detection) and was the only X-ray bright transient in the sample with much fainter X-ray $\nu L_{\nu}$ than that of the optical (shown in Figure 2). Like AT2019pev, the shape of the X-ray rise followed that of the second rise. It was detected by eROSITA as eRASSt J082337+042303, a soft X-ray transient consistent with the galaxy 2MASX J08233674+0423027 (Malyali et al. 2020, 2021). Prior to this, the XMM Slew Survey reported a nondetection at the location of the host galaxy, with an upper limit of $<1.7 \times 10^{-14} \mathrm{erg}^{-1} \mathrm{~s}^{-1} \mathrm{~cm}^{-2}$ assuming $k T_{\mathrm{bb}}=$ $100 \mathrm{eV}$ and $N_{H}=3 \times 10^{20} \mathrm{~cm}^{-2}$. The eROSITA flux of $1.5 \times 10^{-12} \mathrm{erg}^{-1} \mathrm{~s}^{-1} \mathrm{~cm}^{-2}$ was 90 times brighter than this

\footnotetext{
$\overline{23}$ https://cxc.harvard.edu/toolkit/pimms.jsp

${ }^{24} \mathrm{http}: / /$ xmmuls.esac.esa.int/upperlimitserver
}

upper limit. No hard X-ray component was detected above $2.3 \mathrm{keV}$. No strong short-term variability on hours-long timescales was detected, and no strong variability was detected between eROSITA and the three Swift XRT monitoring observations taken afterward with a week-long cadence. Swift and NICER observations over the next 5 months showed an additional increase in X-ray flux by a factor of 10 (Pasham et al. 2020). Pasham et al. (2021) recently reported on additional NICER observations showing high levels of variability on the timescale of days, absorption indicative of a newly launched outflow, as well as a hardening and dimming of the X-ray emission over the course of 8 months. Malyali et al. (2021) report in further detail the X-ray properties of this transient, which showed a net brightening compared to archival data by a factor greater than 600 .

\section{5. $I R$}

A flare in the IR was detected in NEOWISE at the location of AT2019avd 100 days after the discovery of the optical transient and preceding the X-ray transient by approximately 500 days. We present the host-subtracted NEOWISE photometry of the slowly evolving IR flare in Figure 2, and Malyali et al. (2021) presented the non-host-subtracted NEOWISE photometry including data prior to the flare. Prior to this flare, WISE photometry detected no variability at the location of AT2019avd for nearly $5 \mathrm{yr}$.

Malyali et al. (2020) reported that the WISE color of AT2019avd was atypically low ( $W 1-W 2 \simeq 0.07 \mathrm{mag})$ compared to typical AGN values ( $W 1-W 2=0.7-0.8 \mathrm{mag}$; Stern et al. 2012; Assef et al. 2013). The WISE colors of AT2020hle (neoWISE: $0.35 \mathrm{mag}$, AllWISE: $0.036 \mathrm{mag}$ ) and AT2019pev $(W 1-W 2=0.45 \mathrm{mag})$ are also inconsistent with an AGN, though not quite as low as that of AT2019avd. Only AT2019brs truly appeared as an AGN in IR, with WI $W 2=0.98 \mathrm{mag}$. The WISE AGN classification of the sample is summarized in Figure 10 in Section 4.

\subsection{Summary of Preflare Host Galaxy Properties from Archival Observations}

Table 2 presents approximate amplification factors of the flares in the optical, UV, IR, and X-ray wavebands with respect to archival observations for each source in the sample described further in this section.

AT2019brs - This is the only optical transient in this sample spectroscopically identified as an AGN prior to its onset. Rakshit et al. (2017) classified the SDSS spectrum of the host of AT2019brs as that of an NLSy1 galaxy >12 yr prior to the onset of the smoothly flaring transient reported here. Then, the spectrum was not nearly as blue, nor did it display a strong H II emission-line profile. GALEX measured NUV $=21.0 \pm 0.1$ mag for the host prior to 2013. The host also displayed some variability at the $<1 \mathrm{mag}$ level in the Catalina Real-Time Transient Survey (CRTS; Drake et al. 2009) from 2005 to 2013. ROSAT pointed observations measured a flux upper limit of $<1.3 \times 10^{-12} \mathrm{erg} \mathrm{s}^{-1} \mathrm{~cm}^{-2}$ at $0.2-2 \mathrm{keV}$ in 1991 , more than an order of magnitude above the detected flux of the X-ray detection in 2019 reported in Section 2.4.

AT2019pev-GALEX reported a host magnitude of NUV = $18.46 \pm 0.06$ mag from data collected 6 yr prior to the flare. The XMM Slew Survey gave an upper limit at the location of this transient; however, prior to that measurement, it was 
Table 2

Approximate Linear Amplification Factors for Various Wavebands Measured from ZTF Difference Imaging ( $r$ ), Swift UVOT (UVW2), Swift XRT, and NEOWISE (W2), Respectively

\begin{tabular}{lcccc}
\hline \hline Name & Optical & UV & X-ray & IR \\
\hline AT2019pev & 4 & 2 & 100 & 4 \\
AT2019brs & 4 & 7 & $\ldots$ & 10 \\
AT2019fdr & 7 & 6 & $\ldots$ & $>4$ \\
AT2019avd & 2 & 2 & $>600$ & 100 \\
AT2020hle & 2 & $\ldots$ & $\ldots$ & 8 \\
\hline
\end{tabular}

Note. Host levels are measured from SDSS PSF magnitudes ( $r$, GALEX (NUV) and ROSAT, or from reference data when available.

detected in ROSAT (Kara et al. 2019), indicating a likely AGN host. Section 2.4 contains more details on the timing and levels of these archival observations.

AT2019fdr-The GALEX AIS magnitude of the host galaxy was cataloged at NUV $=21.030 \pm 0.306$ mag. ROSAT and the XMM-Newton Slew Survey (Saxton et al. 2008) reported $0.2-2 \mathrm{keV} 3 \sigma$ flux upper limits at the location of this transient between 1990 and 2011 (between 29 and $8 \mathrm{yr}$ prior to the flare) of $<4.6 \times 10^{-13}$ and $<3.1 \times 10^{-12} \mathrm{erg} \mathrm{s}^{-1} \mathrm{~cm}^{-2}$, respectively.

AT2019avd-The NEOWISE light curve of the host remained steadily at the $W 1=12.75$ Vega magnitude level, 1 mag below the maximum flare magnitude, and ASAS-SN difference imaging show no detections for $\sim 2000$ days prior to the optical flare (Figure B.1. of Malyali et al. 2021). Malyali et al. (2021) also report $3 \sigma$ X-ray upper limits between 5 and 30 yr prior to the transient, which showed an increase in 2 $-10 \mathrm{keV}$ luminosity by greater than a factor of 90 . The coadded GALEX magnitude of the host used in Figure 2 is $\mathrm{NUV}=19.22 \pm 0.07$ magnitude

AT2020hle-GALEX measured a host magnitude of $\mathrm{NUV}=20.5 \pm 0.2$. ROSAT and XMM Slew reported six total upper limits for the past three decades including the optical flare, measuring $F_{0.2-2} \mathrm{keV}<2.3 \times 10^{-13} \mathrm{erg} \mathrm{s}^{-1} \mathrm{~cm}^{-2}$ in 1990 and $F_{0.2-2 \mathrm{keV}}<1.2 \times 10^{-12} \mathrm{erg} \mathrm{s}^{-1} \mathrm{~cm}^{-2}$ in 2020 .

\section{Analysis}

\subsection{Photometry}

The difference imaging light curves for the sample are shown in terms of absolute magnitudes in Figure 3.

We show the sample alongside various NLSy1-related events from the literature, which are described in more detail in Section 4. CSS100217 displayed some variability prior to the transient, unlike any of the events in this sample. AT2017bgt was observed only during its fade in difference imaging, so we instead show its aperture photometry (from the ASAS-SN Photometry Database; ${ }^{25}$ Jayasinghe et al. 2019), which also shows the rise of the source. AT2018dyk is by far the least-luminous transient shown. We note the similarity of the shapes of the light curves of AT2019fdr and PS16dtm, which is discussed further in Section 4.

\subsubsection{Light-curve Timescales}

We measured the rise-to-peak timescales of the sample by fitting Gaussians to the light curves shown in Figure 3 using the lmfit package (Newville et al. 2016). We observe a potential

\footnotetext{
25 https://asas-sn.osu.edu/photometry
}

correlation (with a correlation coefficient of $r=-0.56, p=$ 0.08 ) between the luminosity (specifically the absolute magnitudes $M_{V}$ and $M_{g}$ ) and rise-to-peak rest-frame timescales of the sample ( $t_{\text {rise }}$ in days) with the following relation: $M=-0.04 t_{\text {rise }}-19.03$, shown in Figure 4. Fitting the light curves with quadratic functions resulted in the same correlation within the error estimates. Interestingly, TDEs also show a positive correlation between rise time to peak and luminosity, with information about the density and extent of the accreting material imprinted onto this radiative diffusion timescale (van Velzen et al. 2021). Within this framework, AT2018dyk appears underluminous for how fast it rises. AT2017bgt was observed only during its fading phase in difference imaging, and so was excluded from this portion of the analysis.

\subsubsection{Rebrightening}

It is notable that two sources in the sample, AT2019pev and AT2019avd, each have a dramatic rebrightening episode. Following a flare and an approximately $\sim 2$ mag fade from peak, both return to nearly half their maximum luminosity before seasonal gaps in visibility. This is in contrast to that of almost all TDEs and SNe in the literature (e.g., Sollerman et al. 2019, 2020), although they can show plateaus and "humps" (e.g., Hammerstein et al. 2021) ${ }^{26}$ We explore possible interpretations of this rebrightening in Section 4.

\subsubsection{UV/Optical to X-Ray Ratio}

We derive the simultaneous UV/optical-to-X-ray spectral slope ratio $\left(\alpha_{\mathrm{OX}}\right)$ from the Swift UVOT and XRT observations of the sample (as well as upper limits assuming $\Gamma_{X}=2$, when applicable). Following Equation (4) of Tananbaum et al. (1979) and Equation (11) of Grupe et al. (2010), the definition of this ratio is $\alpha_{\mathrm{OX}}=0.3838 \log \left(L_{2 \mathrm{keV}} / L_{2500 \mathrm{~A}}\right)$. Of the transients detected in X-rays, the $\alpha_{\text {Ox }}$ of AT2019pev evolves over 150 days between 1.1 and 1.4, and AT2019brs is observed in $\mathrm{X}$-rays during only one epoch with $\alpha_{\mathrm{OX}}=1.7$, equivalent to that of the late-time detections of AT2019avd. The range of $\alpha_{\mathrm{OX}}$ measured for the sample is consistent with that of NLSy1s $\left(0.9<\alpha_{\text {OX }}<1.8 ;\right.$ Gallo 2006).

\subsection{Spectroscopy}

From the FWHM of the broad Balmer emission lines, we classified all sources in the sample as NLSy1s. We fit the $\mathrm{H} \alpha$ and $\mathrm{H} \beta$ line profiles of the host (when available) and H II $\lambda 4686 \AA$ line profiles of the transient spectra of the sample with the nonlinear least-squares minimization and curve-fitting routine in the lmfit Python package. The results of these fits are shown in Figure 5. Using a Lorentzian profile for the broad $\mathrm{H} \alpha$ component fit provided an improvement of the fit over that of a Gaussian profile, as would be expected based on studies of NLSyls (e.g., Nikołajuk et al. 2009).

We compare the host (when available) and transient spectra of this sample to other transients in NLSy1s in Figure 6 (showing the full wavelength range of the observations) and 7 (rest wavelength $3700-5150 \AA$, showing clearly the He II, Fe II, and $\mathrm{H} \beta$ line profiles). In Figure 7 we color-code the sample (as well as these known NLSy1-related transients in the

\footnotetext{
${ }^{26}$ We note that ASASSN-15lh showed a large-amplitude "double-humped" structure in its UV light curve.
} 

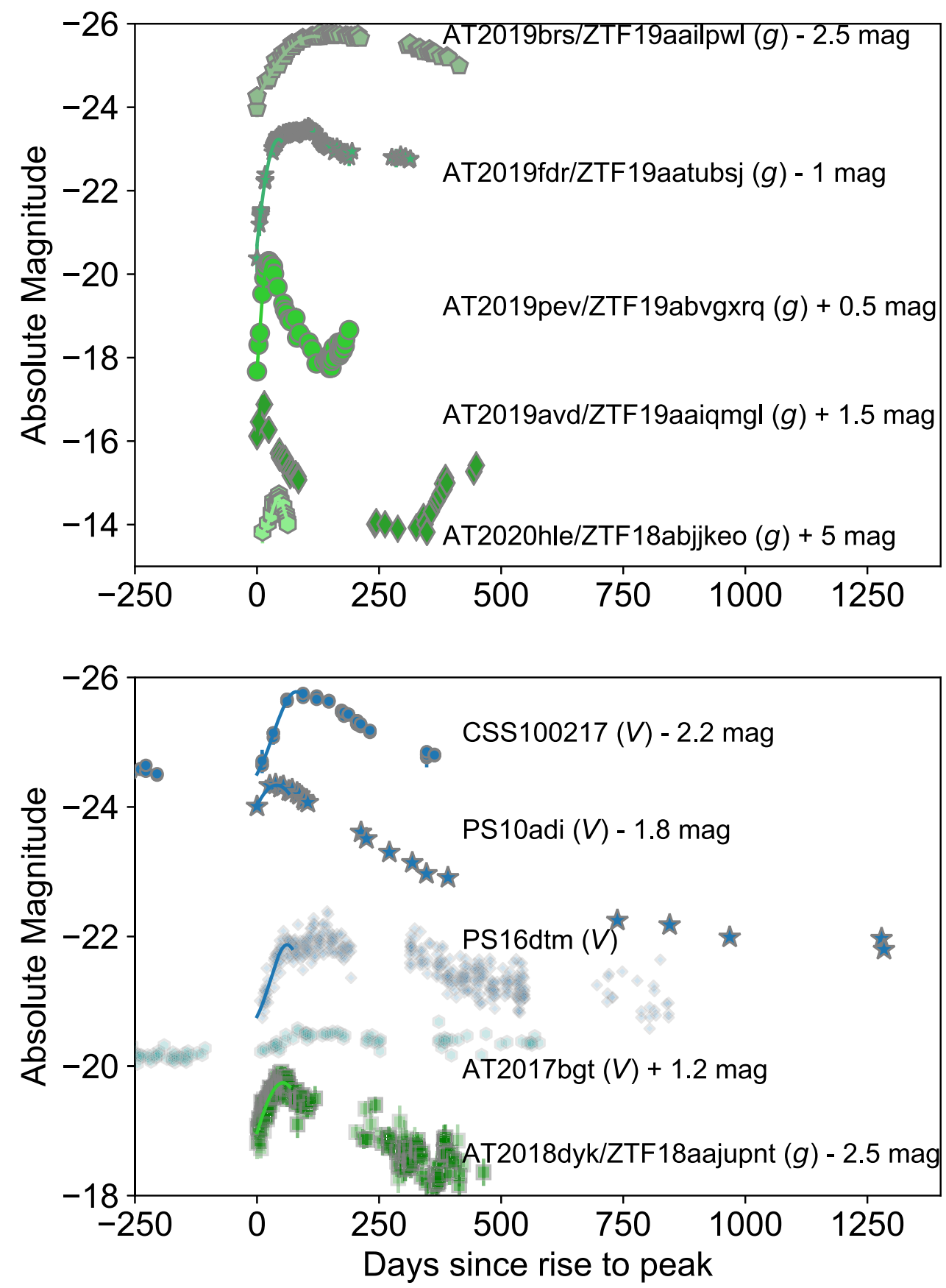

Figure 3. The difference imaging light curves of the ZTF sample (upper panel) compared to the published light curves of NLSy1-related events from the literature (lower panel): changing-look LINER AT2018dyk (Frederick et al. 2019), TDE in NLSy1 PS16dtm (Blanchard et al. 2017), optical transients in NLSy1s CSS100217 (Drake et al. 2011) and PS1-10adi (Kankare et al. 2017), and the aperture photometry of flaring NLSy1 AT2017bgt (Trakhtenbrot et al. 2019a). We show only $g$-band observations for the ZTF sample (upper panel), and omit error bars for visual purposes. Note the differences in optical filters shown ( $g$ in green, $V$ in blue), the differences in colors and markers used to represent the same filters for visual clarity, as well as the difference in $y$-axis scale between the panels. CRTS data for CSS100217 > 200 days prior to the transient are not shown on this scale but showed no significant activity for $>5$ years.

literature) based on the observational classification scheme we establish in Section 4.3, named after the features discussed in the following sections: "H II only", "H II+N III", and "Fe II only.",27

When compared to the newly discovered flaring events to those in the literature, it is clear that AT2017bgt (Trakhtenbrot et al. 2019a) has a much stronger $\mathrm{H}$ II $+\mathrm{N}$ III Bowen

\footnotetext{
27 We note that although "only" is used in the categorization naming based on the presence of spectral features, all have strong Balmer features.
}

fluorescence profile, CSS100217 (Drake et al. 2009) has stronger narrow emission lines overall, and AT2018dyk (Frederick et al. 2019) has a weaker blue continuum. The presence and strength of Fe II are uncorrelated with other spectroscopic properties of the transients shown. Of the ZTF sample, the transient spectrum of AT2019fdrshows the strongest Fe II complex. However, AT2019fdr shows no strong He II + Bowen fluorescence features while the others in the ZTF sample do. AT2019fdr and AT2019avd both show offset blue components of $\mathrm{H} \beta$. 


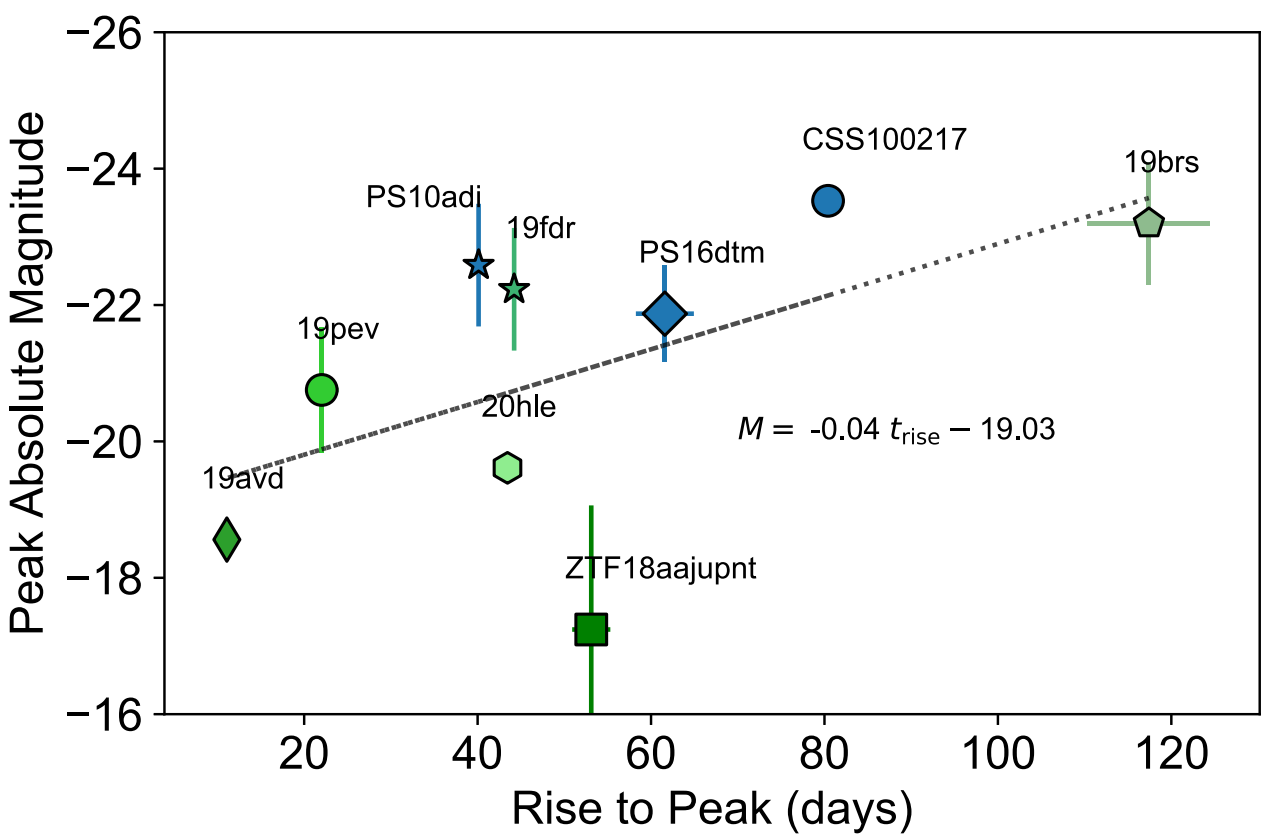

Figure 4. The relation between the rise times of the sample light curves with the maximum absolute magnitude. Fits to light curves are described in Section 3.1.1. The same color scheme and markers are used as in Figure 3.

\subsubsection{Strong He II Profiles in AGNs?}

In the discovery paper for transient ASASSN-18jd, Neustadt et al. (2020) emphasized the relatively rare nature of strong H II emission in AGNs in general, noting the exceptions in the Trakhtenbrot et al. (2019a) observational class of flares as well as the rapid changing-look AGN event AT2018dyk (Frederick et al. 2019). Peterson \& Ferland (1986) reported a direct signature of such transient accretion activity for the first time following a dramatic variability event in the well-studied type 1 Seyfert NGC 5548. The appearance of a H II $\lambda 4686 \AA$ feature broader than the broad-line region (BLR) was delayed approximately 30 days with respect to a continuum flare, which was accompanied by EUV brightening. We report in Table 3 that the $\mathrm{H}$ II lines are broader than that of the Balmer line features, similar to the findings of Peterson \& Ferland (1986) in NGC 5548. They interpreted the broad H II as a feature of a "very broad-line region" closer in to the central SMBH than the BLR. A strong H II line profile is common (but not ubiquitous) in the spectra of TDEs, and they are typically accompanied by Bowen fluorescence features (e.g., Blagorodnova et al. 2019; van Velzen et al. 2021). AT2019avd, AT2019pev, and AT2019brs, with FWHM(He II) reaching $\sim 6500 \mathrm{~km} \mathrm{~s}^{-1}$, look the most similar to AT2017bgt spectroscopically. They are spectroscopically classified as "H II $+\mathrm{N}$ III"-type flares in Figure 7.

\subsubsection{The Fe II complex}

A strong Fe II line complex (blueward and redward of $\mathrm{H} \beta+[\mathrm{O} \mathrm{III]}]$ in optical spectra, between 4434 and $5450 \AA$ ) is a distinguishing feature of NLSy1 galaxies. Reverberation mapping studies of AGNs show that the line-complex-emitting region is measured farther than the Balmer-line-emitting region (e.g., Barth et al. 2013; Rafter et al. 2013). The Fe II complex seen in PS16dtm was interpreted as evidence of the system being an NLSy1 prior to the onset of the flare. CSS100217 also displayed a strong Fe II complex and was interpreted as an SN in an NLSy1 (Drake et al. 2011). TDE AT2018fyk also showed low ionization lines including an Fe II $(37,38)$ emission multiplet emerging for 45 days during the TDE and forms a class of Fe-rich TDEs along with ASASSN-15oi and PTF09ge (Wevers et al. 2019). Therefore, this feature may indicate the presence of an AGN but is not always useful in determining the nature of a particular AGN-related flare. For two of the transients in this sample, whether or not the Fe II complex can be seen in optical spectra depends on the phase and the continuum brightness of the transient-for AT2019fdr it was not observed for 368 days, and for AT2019avd it became no longer visible during the second rise 444 days after the initial spectrum was taken.

\subsection{X-Rays}

The Swift XRT data were collected in photon counting mode and processed ${ }^{28}$ using HEASOFT v6.22 (Evans et al. 2009; NASA High Energy Astrophysics Science Archive Research Center (Heasarc) 2014) ${ }^{29} \mathrm{We}$ assessed best-fit models utilizing $\chi^{2}$ statistics and XSPEC version 12.9.1a (Arnaud 1996). Stated uncertainties are at $90 \%$ confidence intervals.

There are only two significantly X-ray detected transients in the sample: AT2019pev and AT2019avd. We show their X-ray spectra in Figure 8 fit to power-law models. The third, AT2019brs, was only detected in one epoch and not at a level that allowed for the signal-to-noise ratio necessary for a spectrum, at 0.003 counts s$^{-1}\left(L_{2-10 \mathrm{keV}}=1.5 \times 10^{42} \mathrm{erg} \mathrm{s}^{-1}\right)$ on 2019 April 18.

The X-ray spectrum of AT2019pev is measured by Swift XRT with a power-law index of $\Gamma=2.99 \pm 0.02$, typical of the strong soft X-ray excess observed below $1 \mathrm{keV}$ in NLSy1s $(\bar{\Gamma}=2.8 \pm 0.9$; , Boller et al. 1996; Forster \& Halpern 1996; Molthagen et al. 1998; Rakshit et al. 2017). The spectrum of AT2019pev could also be explained by a $150 \mathrm{eV}$ blackbody

\footnotetext{
${ }^{28}$ http://www.swift.ac.uk/user_objects/

29 https://heasarc.gsfc.nasa.gov/docs/software/heasoft/
} 


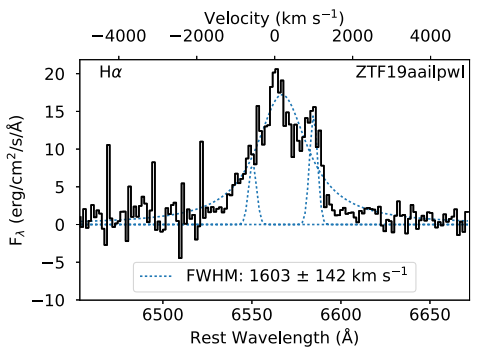

(a) $\mathrm{H} \alpha$ AT2019brs $-13 y$

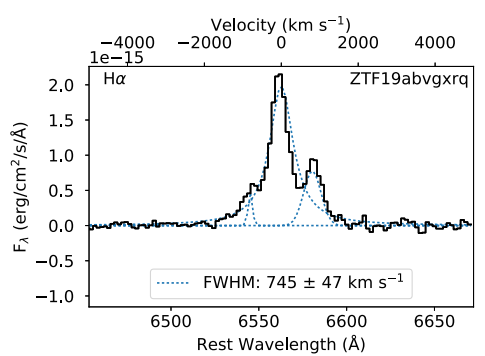

(d) H $\alpha$ AT2019pev Od

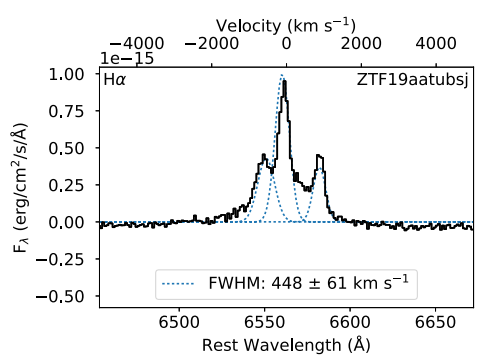

(g) $\mathrm{H} \alpha$ AT2019fdr $+8 \mathrm{~d}$

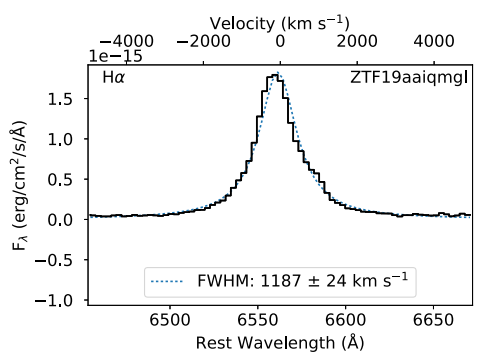

(j) H $\alpha$ AT2019avd +84d

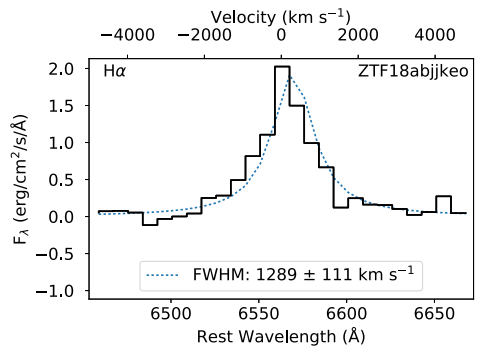

(m) $\mathrm{H} \alpha$ AT2020hle $+8 \mathrm{~d}$

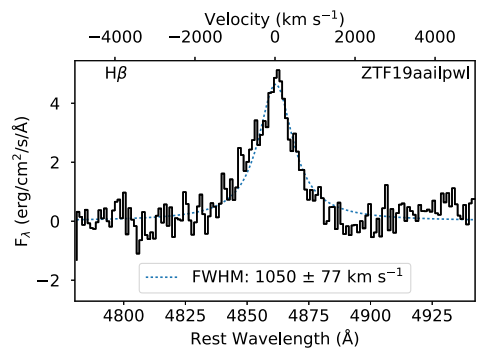

(b) $\mathrm{H} \beta$ AT2019brs -13y

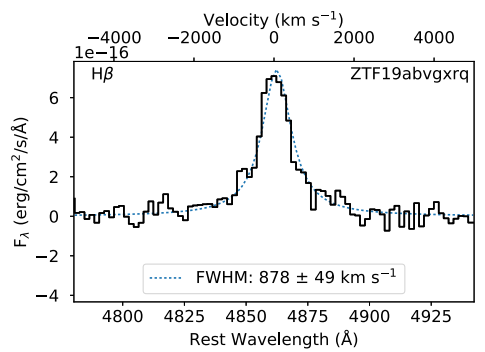

(e) H $\beta$ AT2019pev 0d

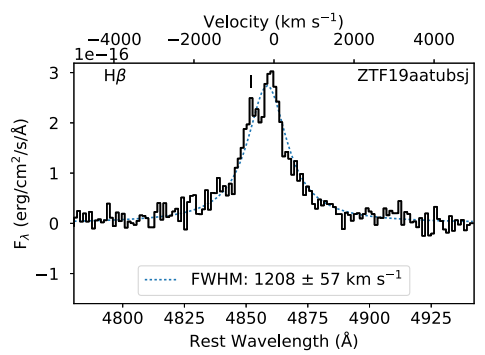

(h) H $\beta$ AT2019fdr $+8 \mathrm{~d}$

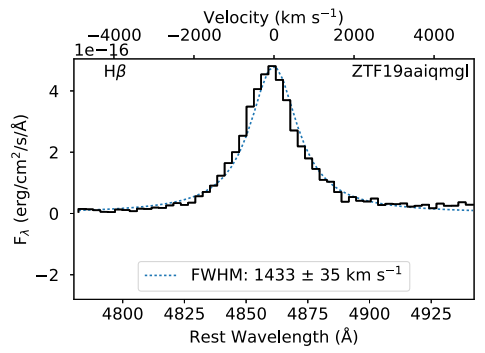

(k) H $\beta$ AT2019avd +84d

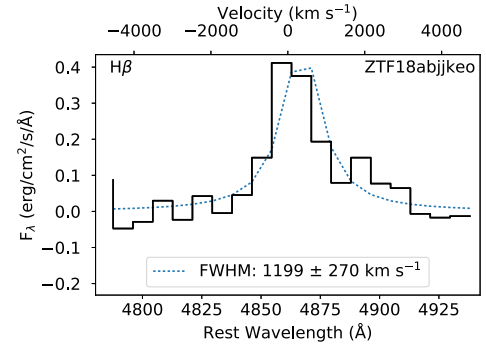

(n) $\mathrm{H} \beta$ AT2020hle $+8 \mathrm{~d}$

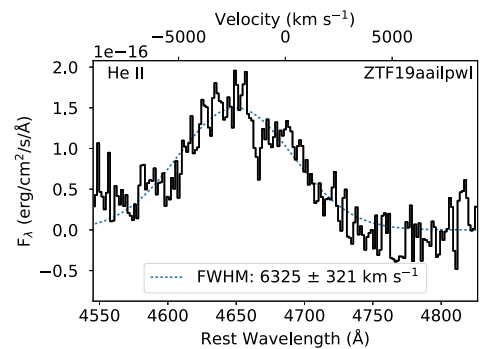

(c) He II AT2019brs +133d

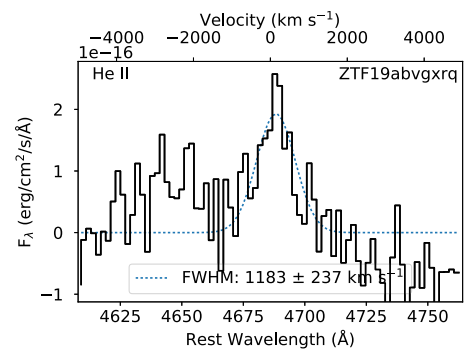

(f) He II AT2019pev 0d

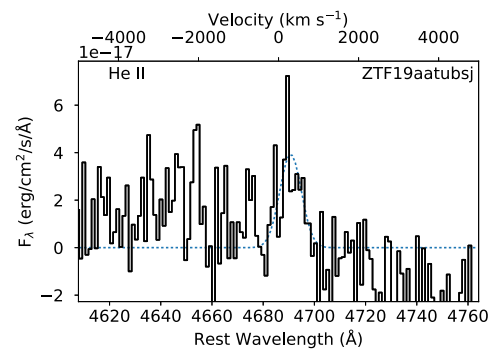

(i) He II AT2019fdr +8d

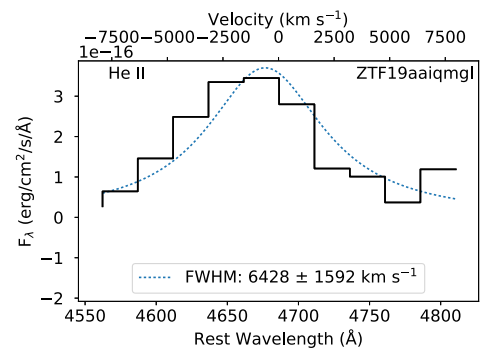

(l) He II AT2019avd +579d

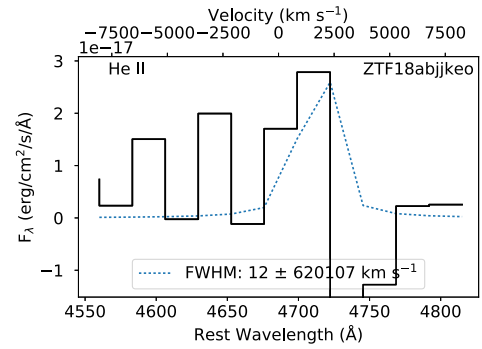

(o) He II AT2020hle +6d

Figure 5. Gaussian fits to the $\mathrm{H} \alpha+[\mathrm{N} \mathrm{II}], \mathrm{H} \beta$, and $\mathrm{H}$ II line profiles of all transients in the sample show that their Balmer lines have an FWHM consistent with (and Lorentzian Balmer profiles characteristic of) that of narrow-line Seyfert $1 \mathrm{~s}$. The offset blue peak in the H $\beta$ profile of AT2019fdr is marked by a vertical line. 


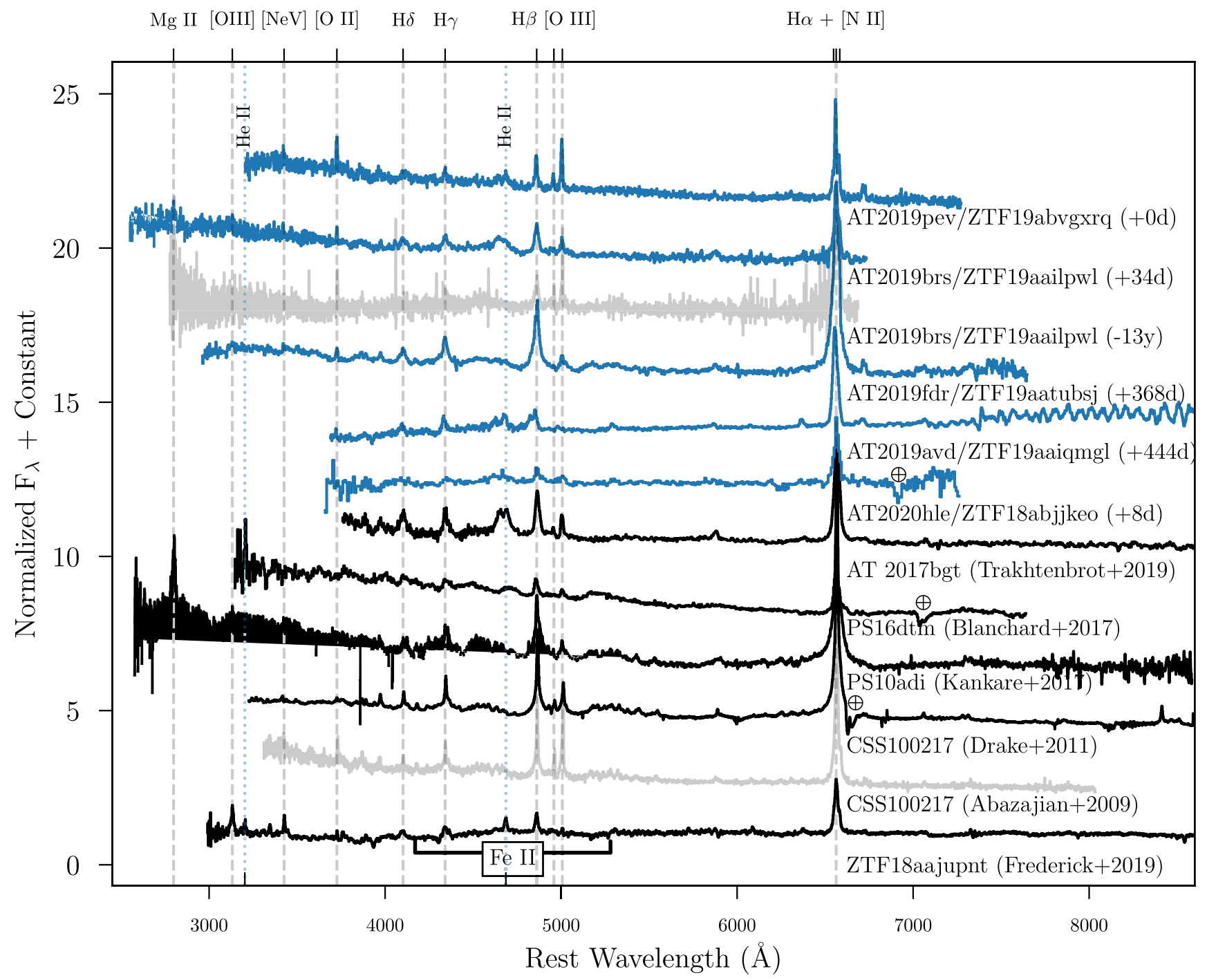

Figure 6. Comparison of the ZTF sample of flares (in blue), as well as discovery spectra for the NLSy1-related events from the literature (in black): changing-look LINER AT2018dyk (Frederick et al. 2019), TDE in NLSy1 PS16dtm (Blanchard et al. 2017), optical transients in NLSy1s CSS100217 (Drake et al. 2011 ) and PS110adi (Kankare et al. 2017), and Bowen fluorescent flare AT2017bgt (Trakhtenbrot et al. 2019a), and their pre-event spectra when available (in gray). For AT2019fdr and AT2019avd here and in Figure 7, we plot the spectra after continuum fading rather than the discovery spectra to display the features used in the spectroscopic classification scheme discussed in Section 4.3. AT2019fdr and AT2019avd show offset blue peaks in H $\beta$, and the peak of H II is offset from $4686 \AA$ in AT2020hle.

with a $\Gamma=2$ power-law component and no intrinsic absorption (Kara et al. 2019). We note that the soft excess observed in NLSy1s can mimic the blackbody temperatures expected for TDEs (e.g., Boller et al. 1996).

The spectral index of AT2019pev $(\Gamma \sim 3)$ was similar to that of AT2018fyk, interpreted as a TDE with late-time disk formation (Wevers et al. 2019), as well as AT2018dyk, interpreted as a changing-look LINER "turning on" into an NLSy1 (Frederick et al. 2019). The X-ray spectral index of AT2019avd was quite high even with regard to these events, with $\Gamma \sim 4-6$.

\subsection{Black Hole Masses and Eddington Ratios}

We measured the BH masses of the sample using two different methods, each with important caveats: the virial mass method, which may systematically underestimate BH masses for NLSy1s, and the host galaxy luminosity, which may be contaminated by the presence of an AGN. The $M_{\mathrm{BH}}$ calculated from the host galaxy luminosity is $M_{\mathrm{BH}, M_{r}}=-0.5 M_{r \text {, host }}-2.96$ following
McLure \& Dunlop (2002), and the standard virial method (e.g., Shen et al. 2011) was employed to obtain the virial BH masses from FWHM $\mathrm{H} \beta$ reported in Table 3.

GWe derive the Eddington ratio for the sample, measuring $L_{\mathrm{bol}}$ at maximum brightness using difference imaging in the optical ( $g$-band or $r$-band) filter with central wavelength closest to $5100 \AA$ in the rest frame and assuming the bolometric correction $L_{\mathrm{bol}}=9 \lambda L_{5100 \mathrm{~A}}$ (Kaspi et al. 2000). Therefore, sources of uncertainty with this method include the dramatic variability in luminosity exhibited during the events illustrated by Figure 2, and that the bolometric correction was not derived empirically from a sample narrow-line Seyfert 1 AGNs (see Runnoe et al. 2012 and references therein for further discussion on uncertainties due to the range of possible bolometric corrections). The transient Eddington ratio estimates depend on the BH masses $\left(M_{\mathrm{BH}}\right)$ as $L_{\mathrm{Edd}}=1.3 \times 10^{38}\left(M_{\mathrm{BH}} / M_{\odot}\right) \mathrm{erg} \mathrm{s}^{-1}$. For each transient in the sample, we report a range of Eddington ratios in Table 3 bracketed by the Eddington ratio measured assuming the virial mass estimate for the $\mathrm{BH}$ mass 


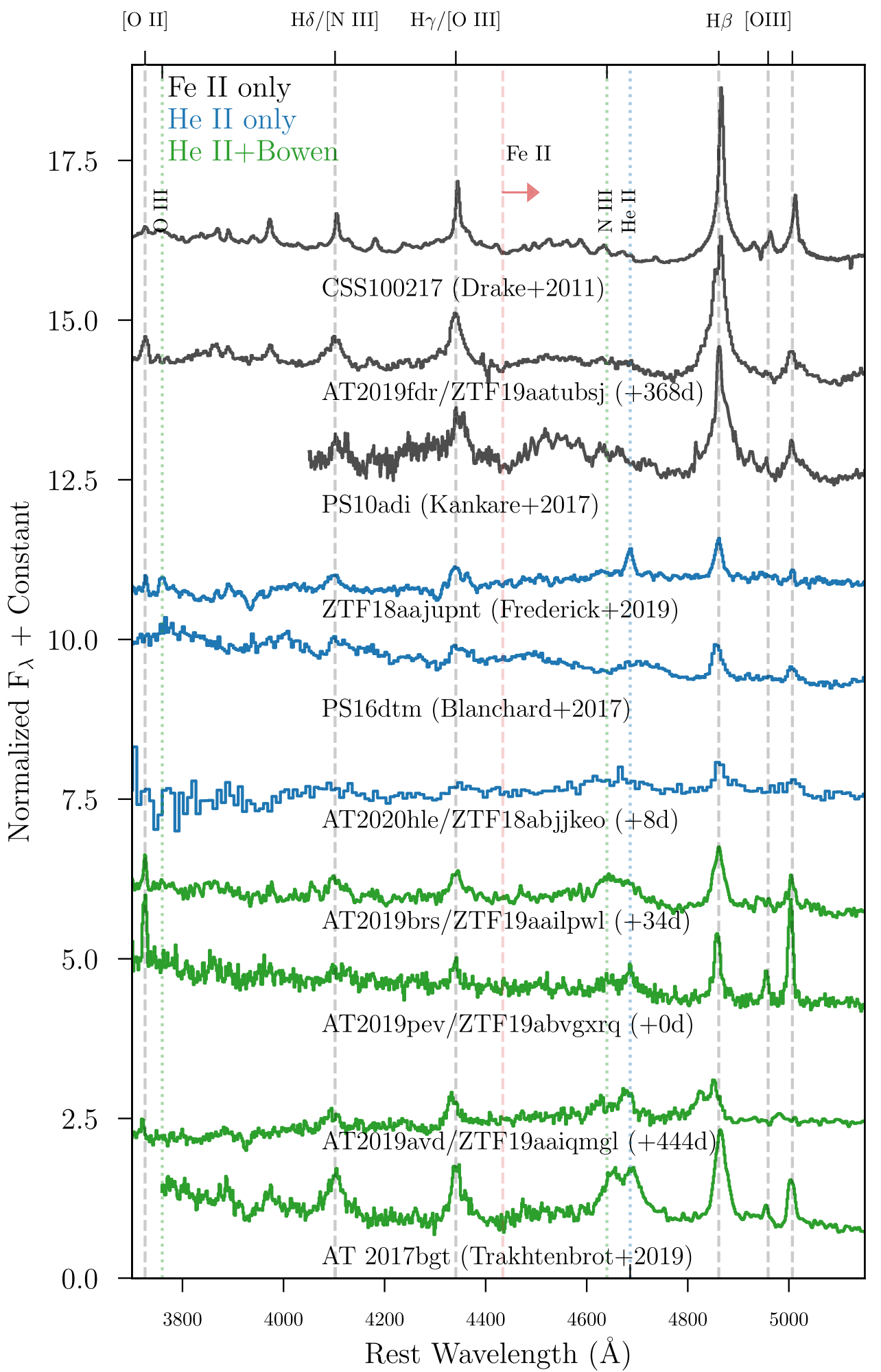

Figure 7. Zoom-in on the 4000-5000 A region of Figure 6 showing the comparison of the strength of $\mathrm{H} \beta$, Fe II, and [O III] of the sample with NLSy1-related events in the literature. We color-code the sample and establish categories based on the presence and absence of key emission line features as described in Section 4.3. Blue spectra indicate the presence of H II, and black spectra indicate transients that displayed Fe II only (though we note that PS16dtm showed both features). Those in green display Bowen fluorescence features in addition to $\mathrm{H}$ II and are most spectroscopically similar to AT2017bgt and the other AGN flares comprising the sample in Trakhtenbrot et al. (2019a).

and the Eddington ratio measured assuming $\mathrm{BH}$ mass derived from the host galaxy luminosity. The range in BH masses, and therefore Eddington ratios, shown in Table 3 is quite large. We estimate statistical and systematic uncertainties of $0.3-0.5$ dex on these mass and Eddington ratio measurements, due to the typical scatter associated with single-epoch mass scaling 


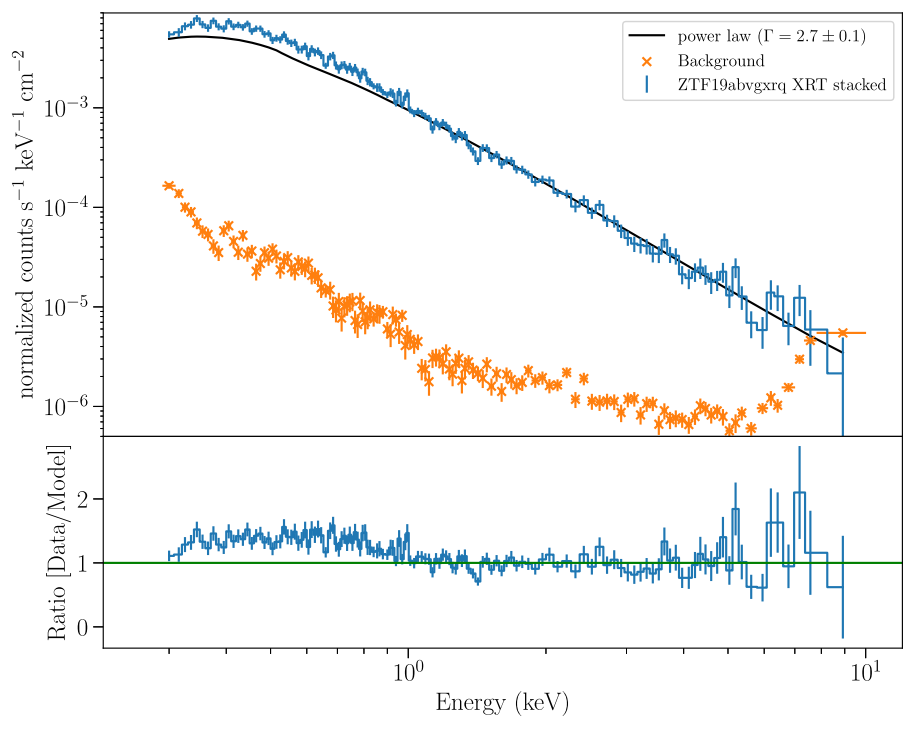

(a) AT2019pev

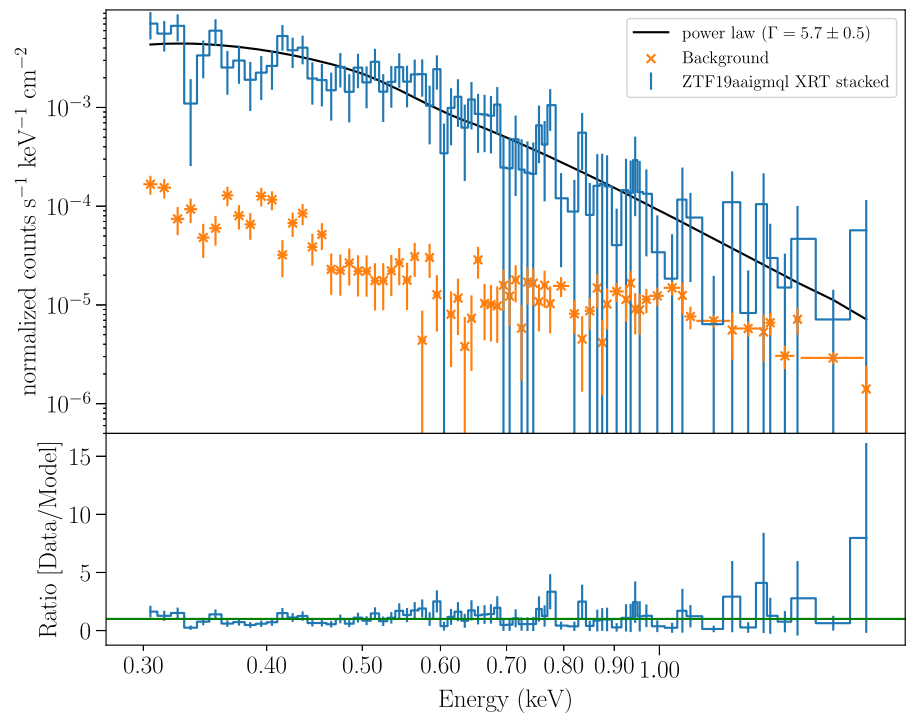

(b) AT2019avd

Figure 8. Left panel: an absorbed power-law fit and ratio residuals to the $\sim 100 \mathrm{ks}$ stacked Swift XRT spectrum of AT2019pev (spectral index $\Gamma=2.7 \pm 0.1$ ). Right panel: the $\sim 4$ ks stacked spectrum of AT2019avd $(\Gamma=5.7 \pm 0.5)$.

Table 3

Black Hole Mass Measurements of the Sample from Optical Spectra and Host Galaxy Properties

\begin{tabular}{|c|c|c|c|c|c|c|c|}
\hline Name & $\begin{array}{l}M_{r, \text { host }} \\
\text { (mag) }\end{array}$ & $\begin{array}{c}\lambda L_{5100 \mathrm{~A}} \\
\left(10^{43} \mathrm{erg} \mathrm{s}^{-1}\right)\end{array}$ & $\begin{array}{c}\mathrm{FWHM}_{\mathrm{H}_{\beta}} \\
\left(\mathrm{km} \mathrm{s}^{-1}\right)\end{array}$ & $\begin{array}{l}\mathrm{FWHM}_{\mathrm{He}} \text { ii } \\
\left(\mathrm{km} \mathrm{s}^{-1}\right)\end{array}$ & $\begin{array}{c}\log M_{\mathrm{BH}, M_{r}} \\
\left(M_{\odot}\right)\end{array}$ & $\begin{array}{c}\log M_{\mathrm{BH}, \mathrm{vir}} \\
\left(M_{\odot}\right)\end{array}$ & $L / L_{\mathrm{Edd}}$ \\
\hline AT2019pev & -21.36 & $5.00 \pm 0.04$ & $878 \pm 49$ & $1183 \pm 237$ & 7.7 & 6.4 & $0.066-1.5$ \\
\hline AT2019brs & -22.38 & $42.6 \pm 0.8$ & $1050 \pm 77^{\mathrm{a}}$ & $6325 \pm 321$ & 8.2 & 7.2 & $0.17-1.97$ \\
\hline AT2019avd & -20.35 & $0.553 \pm 0.008$ & $1433 \pm 35$ & $6428 \pm 1592$ & 7.2 & 6.1 & $0.023-0.29$ \\
\hline AT2020hle & -20.94 & $2.24 \pm 0.02$ & $1199 \pm 270$ & $\ldots$ & 7.5 & 6.4 & $0.048-0.62$ \\
\hline
\end{tabular}

Note. NLSy1s are typically thought to be lower-mass, highly accreting systems, but we show here that the uncertainty in the mass estimates generates significant uncertainty in the estimates of the Eddington ratios (described in Section 3.4). $M_{r \text {,host }}$ is the $r$-band de Vaucouleurs and exponential disk profile model fit magnitude from the SDSS DR14 photometric catalog. The host of AT2020hle is not in the SDSS footprint, and so we instead use the Pan-STARRS1 $r$-band Kron magnitude of this source (Chambers et al. 2016).

${ }^{a}$ The $\operatorname{FWHM}(\mathrm{H} \beta)$ for AT2019brs agrees with the measurement in Rakshit et al. (2017) within the error estimates.

relationships as well as the unknown BLR geometry (e.g., Merloni et al. 2015; Runnoe et al. 2016; Liu et al. 2018a, 2020).

Miller et al. (2019) obtained an independent measurement of the $\mathrm{BH}$ mass of AT2019pev. They measured $M_{\mathrm{BH}}=3.7 \times$ $10^{6} M_{\odot}$ from the observed Chandra X-ray luminosity (this observation is described in more detail in Section 2.4). This is closer to, but not consistent with, the virial mass estimate, meaning that the transient may not have been accreting near the Eddington limit at the time of the X-ray observation.

\section{Discussion}

In this section, we rule out possible physical scenarios for each outburst, beginning with core-collapse SNe IIn. We review why the $\mathrm{SN}$ interpretation was quickly ruled out in favor of an SMBH accretion scenario and discuss how many of the characteristics of the objects are consistent with both NLSy1s and TDEs. We compare the available evidence with other scenarios including TDEs, extreme AGN variability, and binary SMBHs in detail. We also discuss NLSy1 galaxies as the preferential hosts for these and other similar events and outline a scheme for classifying future events based on the presence of spectral features.

\section{1. “IIn or Not IIn?”: Preliminary Observational Classification of the Flare Sample}

Identification of the sample presented here occurred with a slew of conflicting preliminary classifications at early times, which we describe below.

The narrow emission lines in the spectra of some SLSN (Type IIn) are a result of the highly luminous interaction of SN ejecta from a massive progenitor with dense circumstellar medium. Therefore, under special circumstances, nuclear $\mathrm{SNe}$ can look spectroscopically very similar to rapid $^{30}$ flares from NLSy1s in the optical (e.g., Moriya et al. 2018). The shapes of the light curves of the transients in this sample looked rather like those of such SNe, in the absence of additional observations. The smoothness of the flares in particular was unique with respect to typical stochastic AGN variability, and made these transients noteworthy for allocation of follow-up resources. Therefore, the narrow Balmer features in the spectra

\footnotetext{
${ }^{30}$ With rise times on the order of days to weeks.
} 


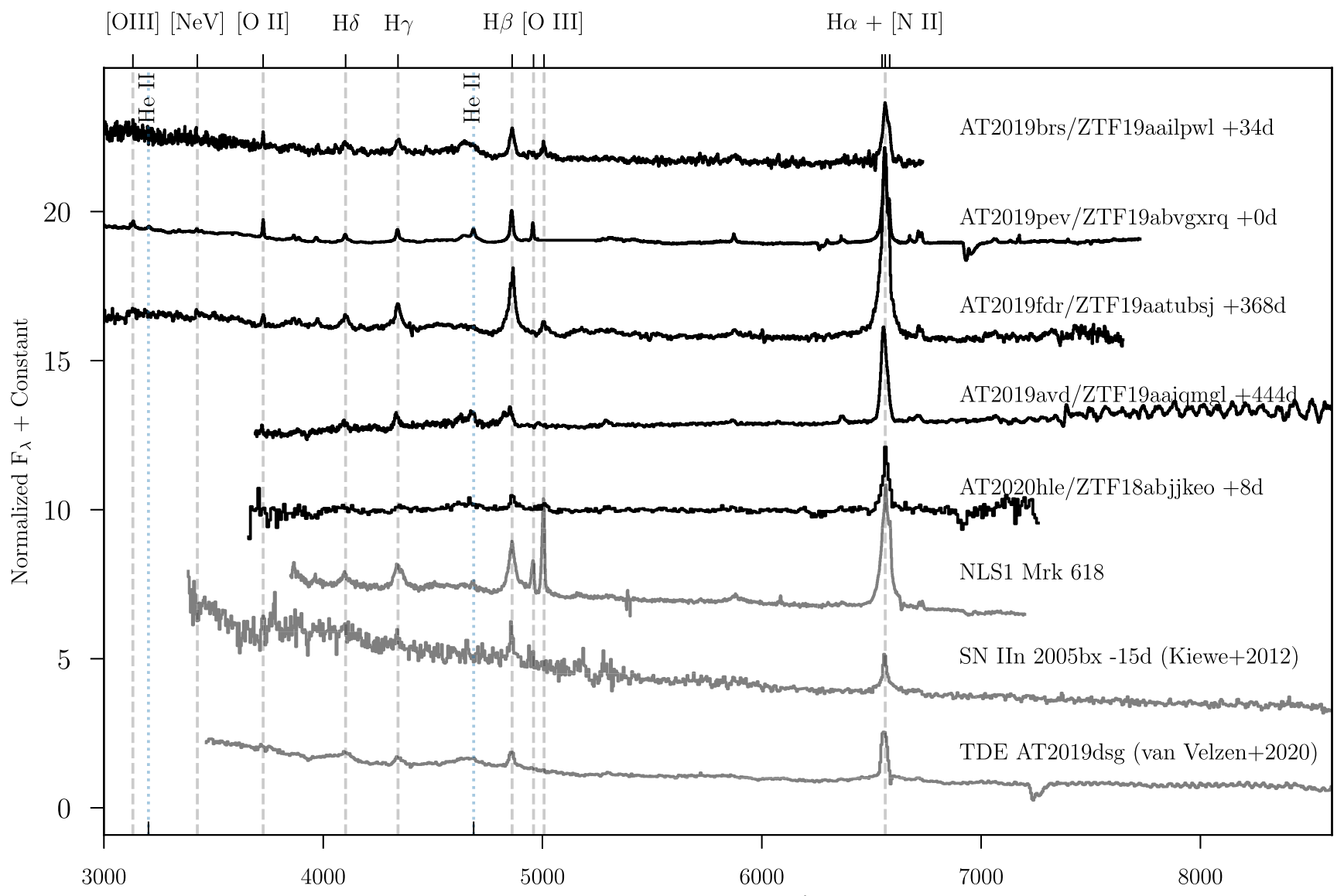

Rest Wavelength $(\AA)$

Figure 9. We compare the spectra of the transient sample (in black) to archetypal NLSy1 Mrk 618, as well as a normal Type IIn SN, SN 2005bx (Kiewe et al. 2012), and AT2019dsg, a normal TDE in a star-forming galaxy with Bowen fluorescence features and a coincident neutrino detection (Stein et al. 2021; van Velzen et al. 2021), in gray.

of these transients, coupled with their light-curve shapes, left uncertainty in their early classifications. They could have been either Type IIn SNe or NLSy1 AGN, while those with persistent strong $\mathrm{H}$ II $\lambda 4686$ features in their spectra looked similar to that of TDEs. To illustrate this, Figure 9 shows spectra of the sample alongside a Type IIn SN as well as a TDE with Bowen fluorescence features. Additional follow-up observations in the UV/X-rays helped distinguish this sample of transients from $\mathrm{SNe}$.

\subsection{A Preponderance of Rapid Optical Transients in Narrow- line Seyfert 1 Host Galaxies}

In the Analysis section (Section 3), we compared our sample to data from nuclear transients in the literature that happened to be hosted in NLSy1 galaxies. In this and the next sections, we discuss NLSy1s as an interesting AGN subtype and observationally classify and link these events to one another on the basis of their shared host properties.

The narrower broad-line Balmer profiles and high-amplitude variability (especially in the X-rays, e.g., Pogge 2000; Frederick et al. 2018) in NLSy1s may be evidence of smaller BH masses in these systems $\left(5<\log \left(M_{\mathrm{BH}}\left[M_{\odot}\right]\right)<8\right.$; e.g., Mathur et al. 2001), and/or higher observed accretion rates (Pounds et al. 1995; Wang et al. 1996; Marconi et al. 2008; Grupe et al. 2010; Xu et al. 2012). The virial masses derived from spectral measurements of the population may also be explained with geometrical effects, when interpreted as the classic broad-line AGNs seen along a lower inclination angle between the broad-line-emitting region and the line of sight (Decarli et al. 2008; Baldi et al. 2016; Rakshit et al. 2017).

Studies of NLSy1s typically find them to be highly photometrically variable only in the X-rays. At optical wavelengths, however, Klimek et al. (2004) found that rapid, high-amplitude variability was rare in a sample of 172 observations of NLSy1s across 33 nights. Ai et al. (2010) also found that NLSy1s had systematically lower optical variability amplitudes $(\lesssim 0.2 \mathrm{mag})$ than broad-line Seyfert $1 \mathrm{~s}$ in a sample of $275 \mathrm{AGN}$ at $0.3<z<0.8$ in $3 \mathrm{yr}$ of SDSS data.

However, optical flares are not unheard of in NLSy1s (e.g., NGC 4051; Guainazzi et al. 1998; Uttley et al. 1999). Klimek et al. (2004) noted the exception of IRAS 13224-3809, which showed both dramatic X-ray and optical variability on short timescales (Miller et al. 2000). Here we describe a number of distinct events, including the Trakhtenbrot et al. (2019a) observational class of optical flares, the "on" state of AT2018dyk, and the host of PS16dtm and CSS100217:102913+404220, which were all consistent with NLSy1-related activity.

Trakhtenbrot et al. (2019a) established a new observational class of dramatic AGN flares accompanied by Bowen fluorescence features. The three events in this class all originated from active BHs that were classified as NLSy1 galaxies by their Balmer FWHMs. Their optical spectra were unusual for NLSy1s in that they showed strong "doublepeaked" H II profiles with contributions from the N III $\lambda 4640$ 
Bowen fluorescence feature, indicating the presence of a strong EUV $(>50 \mathrm{eV})$ ionizing continuum. This was consistent with the UV brightness observed in the small sample of objects as well as the steep blue continua in these sources. The slow UV and spectral emission line evolution over a period of $\sim 450$ days ruled out a TDE, and these were instead interpreted as enhanced accretion onto the SMBH of a preexisting AGN. AT2017bgt was presented as the prototype of these dramatic SMBH UV/optical flares irradiating the BLR. It showed a very slow decrease in optical flux over several months following a relatively shallow ( $\sim 0.5 \mathrm{mag}$ ) rise to peak over $\sim 80$ days from a previous nonvariable state. During the transient, the X-rays increased by a factor of 2-3 from a previous measurement by ROSAT. The persistence of the UV emission over 500 days distinguished it from $\mathrm{SNe}$, and the extremely intense nature of the UV continuum, as well as the presence of the Bowen fluorescence features in the optical spectrum, distinguished it from changing-look AGNs. Two other NLSy1s, OGLE17aaj (Gromadzki et al. 2019) and ULIRG F01004-2237 (Tadhunter et al. 2017) (the latter previously interpreted as a TDE), were retroactively reclassified as belonging to this new observational class of NLSy1s.

Kankare et al. (2017) reported several of a new population of slowly, smoothly evolving nuclear transients with persistent narrow emission lines and strong Fe II. The most energetic and best studied of this sample was PS1-10adi. They presented characteristics of these events indicative of shock interaction, possibly from a new variant of TDE. Jiang et al. (2019) followed up PS1-10adi 1500 days postpeak and reported latestage high-amplitude optical rebrightening as well as a mid-IR flare, putative evidence for a dust echo resulting from the TDE event.

We also compare with AT2018dyk, a changing-look AGN that transformed from a LINER galaxy to an NLSy1. It was identified as such primarily based on X-ray and UV spectra. It displayed strong high-ionization forbidden (i.e., "coronal") emission in the optical and UV spectra, an X-ray flare delayed by 60 days and showed a late-time $g-r$ color change as it faded slowly over $1 \mathrm{yr}$. This was the only AGN with Balmer lines consistent with an NLSy1 among a new class of "changing-look LINERs," including SDSS 1115+0544 (Yan et al. 2019).

PS16dtm (iPTF16ezh/SN 2016ezh) was a near-Eddington but X-ray-quiet nuclear transient with strong Fe II emission and $T_{\mathrm{BB}} \sim 1.7 \times 10^{4} \mathrm{~K}$. It rose over $\sim 50$ days to "superluminous" levels $\left(\log L_{\mathrm{bol}}\left[\mathrm{ergs} \mathrm{s}^{-1}\right]>44\right)$ at peak before plateauing twice over $\sim 50$ and $\sim 100$ days while maintaining a constant blackbody temperature. The event was interpreted as a TDE exciting the BLR in a well-studied, spectroscopically confirmed NLSy1 with $M_{\mathrm{BH}} \sim 10^{6} M_{\odot}$ (Blanchard et al. 2017). X-ray upper limits showed dimming by at least an order of magnitude compared to archival observations, but Blanchard et al. (2017) predicted the X-rays would reappear after the obscuring debris (oriented perpendicularly to the accretion disk) had dissipated. We show the $V$-band ASAS-SN photometry for PS16dtm in Figure 4, which appears similar in shape and absolute magnitude to AT2019fdr, though longer in duration.

CSS100217:102913+404220 displayed a high state $\left(M_{V}=\right.$ -22.7 at 45 days postpeak) accompanied by broad $\mathrm{H} \alpha$ and was interpreted either as a Type IIn SN (Drake et al. 2011) or a TDE (Saxton et al. 2018) near the nucleus ( $150 \mathrm{pc})$ of an NLSy1 in a star-forming galaxy. It eventually faded back to slightly below its original level after one year, which was interpreted as interacting with and subsequently flushing a portion of the accretion disk.

Similar events are not unheard of in broad-line AGN systems, though they may be comparatively rarer. Neustadt et al. (2020) reported a candidate for such a rapidly flaring event with quasar-like properties, ASASSN-18jd, although continued observations of this transient will be critical for a better understanding of the properties of the host.

\subsection{Observational Classification: The "Family Tree" of NLSy1-associated Transients}

In Figure 10, we use this sample to motivate a framework for quickly classifying similarly ambiguous flaring events. We investigate the following:

1. AGN/NLSy1 characteristics: 1) an empirical $W 1-W 2$ WISE color cutoff from Stern et al. (2012), and Assef et al. (2013), which is comparable between NLSy1s and broad-line Seyfert 1s (Chen et al. 2017); 2) a strong Fe II complex; 3) narrow Balmer emission; and 4) [O III]/H $\beta<3$ (Rakshit et al. 2017),

2. TDE characteristics: 1) host central SMBH mass below the Hills mass $\left(\sim 10^{8} M_{\odot}\right)$, and 2 ) a lack of cooling or 3) significant rebrightening,

3. X-ray properties, the presence of which can occur in both AGN and TDEs, but are less likely in the SN scenario.

We apply these criteria in Figure 10 and color-code them as blue or green based on whether they favor the TDE or AGN scenario, respectively (as the SN scenario has been ruled out in Section 4.1). The spectroscopic class, based on the presence of N III Bowen fluorescence features, Fe II, and/or H II $\lambda 4686$, which can occur in both TDEs as well as flaring NLSy1s, is then interpreted in the context of one of these scenarios. Based on this table, we confirm the interpretations for three of the four NLSy1-associated transients reported in the literature, except for CSS100217, for which we favor the AGN scenario over the SN interpretation.

Summarized briefly: We expect that transients with strong Fe II complexes are most likely associated with AGN, those with very steep soft X-ray spectra $(\Gamma>5)$ and no intrinsic absorption are most likely associated with TDEs, and those with strong Bowen fluorescence profiles and slow UV and spectral evolution are likely associated with enhanced accretion onto SMBHs from a preexisting accretion disk. The timing of a mid-infrared flare may also help to distinguish between an AGN and a TDE - if it precedes the optical, it is likely associated with AGN variability, but if it follows as an echo, it may be associated with a TDE (van Velzen et al. 2016).

van Velzen et al. (2021) established a spectroscopic classification scheme for the sample of TDEs discovered during the first half of the ZTF survey, distinguishing those with and without $\mathrm{H}$ II in a single epoch. About half of the TDEs in that sample were "H only", and only one was "He only". They found that higher density conditions were likely for the rest of the TDEs which had $\mathrm{H}$ and He lines, as well as Bowen features.

For the flaring NLSyl sample presented here, we establish the following spectroscopic classes to describe each of the transients based on the presence or absence of emission features crucial to their physical interpretations: 


\begin{tabular}{|l|c|c|c|c|c|c|c|c|c|c|c|c|}
\hline Name & $\begin{array}{c}\log M_{\mathrm{BH}}<8 \\
{\left[M_{\odot}\right]}\end{array}$ & $\begin{array}{c}\mathrm{H} \beta<2000 \\
\mathrm{~km} \mathrm{~s}^{-1}\end{array}$ & Fe II & $\begin{array}{c}{[\mathrm{OIII}] / \mathrm{H} \beta<3} \\
{[\text { flux ratio] }}\end{array}$ & $\begin{array}{c}\Delta g-r \\
\sim 0 \mathrm{mag}\end{array}$ & $\mathrm{UV}$ & X-ray $\Gamma$ & $\begin{array}{c}\text { W1-W2 } \\
>0.7 \text { mag }^{\mathrm{a}}\end{array}$ & $\begin{array}{c}\text { Re- } \\
\text { brighten }\end{array}$ & Spec. class & Interp. \\
\hline AT2019brs & $\times$ & $\checkmark$ & $\checkmark$ & $\checkmark$ & $\times$ & $\checkmark$ & $\checkmark$ & $\checkmark$ & $\times$ & HeII+NIII & AGN \\
AT2019pev & $\checkmark$ & $\checkmark$ & $\times$ & $\checkmark$ & $\checkmark$ & $\checkmark$ & 3 & $\times$ & $\checkmark$ & HeII+NIII & AGN \\
AT2019fdr & $\checkmark$ & $\checkmark$ & $\checkmark$ & $\checkmark$ & $\times$ & $\checkmark$ & $\times$ & $\times$ & $\times$ & FeII & TDE \\
AT2019avd & $\checkmark$ & $\checkmark$ & $\checkmark$ & $\checkmark$ & $\times$ & $\checkmark$ & 5 & $\times$ & $\checkmark$ & HeII+NIII & AGN \\
AT2020hle & $\checkmark$ & $\checkmark$ & $\times$ & $\checkmark$ & $\checkmark$ & $\checkmark$ & $\checkmark^{\mathrm{b}}$ & $\times$ & $\times$ & HeII & TDE \\
\hline CSS100217 & $\checkmark$ & $\checkmark$ & $\checkmark$ & $\checkmark$ & $\times$ & $\checkmark$ & 3 & $\checkmark$ & $\times$ & FeII & AGN \\
PS16dtm & $\checkmark$ & $\checkmark$ & $\checkmark$ & $\checkmark$ & $\checkmark$ & $\checkmark$ & $2^{\mathrm{c}}$ & $\times$ & $\times$ & HeII+FeII & TDE \\
AT2017bgt & $\checkmark$ & $\checkmark$ & $\checkmark$ & $\checkmark$ & $\checkmark$ & $\checkmark$ & 2 & $\times$ & $\checkmark$ & HeII+NIII & AGN \\
AT2018dyk & $\checkmark$ & $\checkmark$ & $\times$ & $\checkmark$ & $\times$ & $\checkmark$ & 3 & $\times$ & $\times$ & HeII & AGN \\
PS1-10adi & $\checkmark$ & $\checkmark$ & $\checkmark$ & $\checkmark$ & $\checkmark$ & $\checkmark$ & $\checkmark^{\mathrm{b}}$ & $\times$ & $\times$ & FeII & TDE \\
\hline
\end{tabular}

Figure 10. Comparison of the properties of individual objects in the sample (upper table) and NLSy1-related transients in the literature (lower table). " $\checkmark$ " means that the property is observed, and " $\times$ " indicates that the characteristic was not observed. "UV" refers to the persistence of detected UV emission and "Rebrighten" refers to a significant recovery of at least half the peak luminosity of the source. Following the convention of Figure 7, blue (green) indicates a property associated with the TDE (flaring AGN) scenario. a. We select the less conservative color cut presented in Stern et al. (2012). b. The single low-level XRT detection of AT2019brs and AT2020hle occurred only once throughout the follow-up campaign and was not at a level to take a reliable spectral measurement. Similarly, the late-stage X-ray detection of PS10adi reported in Jiang et al. (2019) was not sufficient to measure the softness of the spectrum. c. The host of PS16dtm displayed X-rays only prior to and following the fading of, but not for the duration of, the transient.

1. "H II only",

2. "H II+N III", and

3. "Fe II only",

and we propose the following naming convention for these classes: "NLSy1-He II", "NLSy1-He II+N III," and "NLSy1Fe II". 31

\subsection{Physical Interpretation of the Transient Flares}

In the following section we consolidate all that is known about the relevant properties of each object in the sample and compare them with the related transients in NLSy1s in the literature to explore each of the following scenarios: a PS16dtm-like TDE in an NLSy1, a Sharov 21-like microlensing event, a CSS100217-like SN in an NLSy1, and a binary SMBH scenario.

\subsubsection{Association of the Transients with AGNs}

There is evidence that all sources in the sample are associated with AGNs rather than distinct explosive events occurring in a normal galaxy. As evident in Figure 9, the strengths of the Balmer lines in the transient spectra are most consistent with that of an NLSy1. Ne V $\lambda 3426$, when observable, is typically associated with AGNs and is present in the spectra of these sources. Strong H II profiles, although somewhat rare in association with normal stochastic AGN variability (Neustadt et al. 2020), have been observed before and interpreted as the signature of a sudden enhancement of accretion (e.g., Frederick et al. 2019; Trakhtenbrot et al. 2019a).

Persistent X-rays are a likely signature of accretion onto an SMBH rather than an SN. A strong soft X-ray excess is characteristic of NLSy1s. However, it is typically accompanied by a hard X-ray continuum component (not present in either

\footnotetext{
$\overline{31}$ We note that although hydrogen features are not explicitly named in this feature classification scheme, all spectra of the transients show resolved narrow $\left(1000<\right.$ FHWM $<2000 \mathrm{~km} \mathrm{~s}^{-1}$ ) Balmer features (see Section 3.2).
}

$\mathrm{X}$-ray-detected transient in this sample) and not nearly as ultrasoft as the X-rays seen in AT2019avd $(4 \lesssim \Gamma \lesssim 6)$, which are slopes more frequently observed in the $\mathrm{X}$-ray spectra of TDEs.

Although these outbursts may not necessarily be the result of an intrinsic enhancement in AGN accretion activity, transients with fast-rise/slow-decay (such as those in this sample), along with slow-rise/fast-decay and symmetric light-curve shapes, were well represented in a sample of 51 AGN flares discovered in CRTS (Graham et al. 2017). When taken together, these light-curve shapes can be connected to the ongoing physical and radiative interactions during these accretion events, similarly to TDEs (van Velzen et al. 2021).

\subsubsection{The SN Scenario}

It is highly improbable that these flares are the result of normal SN explosions. We observe long-lived $U$-band emission in AT2019fdr, persistent UV emission in all transients in the sample, and strong transient X-ray detections in AT2019pev and AT2019avd. There is also only a small likelihood of an SN in the host galaxy along the line of sight unassociated with the AGN. The strongest evidence against the normal SN scenario is the persistence of the H II emission features $\sim 10-100$ days after the onset of the flare-such flash ionization signatures are only visible in SN spectra at very early times (e.g., Khazov et al. 2016; Bruch et al. 2021).

At least one of these transients (AT2019fdr) shares a number of properties with CSS100217, which displayed soft X-rays and was interpreted as an SN IIn explosion in an AGN disk. The SN interpretation of CSS100217 was largely based on light-curve energetics, which are similar to those of this sample. The $g-r$ color change and the peak magnitude of $-23 \lesssim M_{V} \lesssim-22$ are very similar in particular between CSS100217 and AT2019fdr. Type IIn SNe can exhibit strong Fe II lines in late spectra, such as AT2019fdr did.

However, in contrast, the light-curve evolution differs in that CSS100217 fades at least twice as quickly as AT2019fdr. Also, the Fe II complex of CSS100217 was always visible throughout 
the flare, and Drake et al. (2011) observed a broad $\sim 3000 \mathrm{~km} \mathrm{~s}^{-1}$ component in $\mathrm{H} \alpha$ which got broader with time in subsequent follow-up spectra of CSS100217. Strong P Cygni profiles are observed in the optical spectra of SN, and from such profiles, we would expect an absence of absorption on the blue end of the Balmer line profiles, rather than emission as in the spectra of AT2019avd and AT2019fdr. Therefore, based on this evidence we rule out the SN Type IIn scenario.

\subsubsection{The TDE Scenario}

None of the events in this sample are consistent with the set of characteristics typically displayed by an isolated tidal disruption occurring in a dormant SMBH system. We therefore focus on the possibility for TDEs in preexisting AGNs as in Merloni et al. (2015) and Chan et al. $(2019,2020)$ and compare features of the events in our sample to observations of such TDEs as well as studies of standard TDEs in the literature, although we acknowledge the possibility for more exotic TDE scenarios such as those explored for AT2019avd in Malyali et al. (2021).

The Hills mass is the mass for which the tidal Roche radius is equivalent to the gravitational Schwarzschild radius of the $\mathrm{BH}$, beyond which a star (that would otherwise be tidally pulled apart) is instead left intact as it passes the event horizon (Hills 1975). This maximum mass to tidally disrupt a solar-type star just outside the event horizon is $10^{8} M_{\odot}$. Therefore, an SMBH mass significantly above this limit would likely rule out a TDE. Of the SMBH masses derived for the host galaxies, only that of AT2019brs is inconsistent with a TDE scenario (although we note that it is consistent within the typical uncertainty for such mass measurements). The range of absolute magnitudes of the flares in this sample $(-23<$ $M_{r}<-19 \mathrm{mag}$ ) also tend to be intrinsically brighter at peak than all but one of the ZTF TDEs $\left(M_{r}>-20 \mathrm{mag}\right)$ reported in van Velzen et al. (2020), AT2018iih $\left(M_{r}=-21.5 \mathrm{mag}\right)$.

Similar to TDEs PS16dtm and AT2018fyk, AT2019fdr showed two distinct plateau stages on month-long timescales after fading, with some slight fading in between. Color evolution is rare but not unheard of for TDEs, and the cooling AT2019fdr shows postpeak is slow, with the transient still detected in the UV at late times as would be expected for a TDE. Optical rebrightening following the initial flare has been interpreted as the result of late-time disk formation in a number of TDEs (e.g., van Velzen et al. 2019; Wevers et al. 2019). However, rebrightening with high amplitudes returning nearly to preflare levels such as that seen in AT2019pev and AT2019avd has neither been observed ${ }^{32}$ nor predicted (e.g., Chan et al. 2019, 2020) from a TDE. In these cases with rebrightening, a TDE is strongly ruled out.

AT2019avd and AT2019fdr, like AT2018fyk, only showed Fe II at certain times during the flare. AT2019avd only displayed Fe II during its first peak, and in AT2019fdr, the Fe II complex got more visible as the transient faded. AT2019fdr is the only transient in the sample with a lack of H II features in its spectra. Within the van Velzen et al. (2021) spectral classification scheme for optical TDEs, AT2019fdr would be a H-only TDE, with the Fe II complex attributed to the NLSy1 host. It is important to note that the transients with blue horn features in $\mathrm{H} \beta$, AT2019avd and AT2019fdr, may be

\footnotetext{
${ }^{32}$ Except in the case of the periodicity of ASASSN-14ko, which was interpreted as a possible repeating partial TDE (Payne et al. 2021).
}

signatures of wind ejecta with a velocity distinct from the AGN.

Enhanced N III lines such as those seen in the NLSy1-He II +N III spectroscopic class (AT2019pev, AT2019brs, and AT2019avd) are a prediction of TDEs in AGNs when compared to the host spectrum (Kochanek 2016; GallegosGarcia et al. 2018; Liu et al. 2018b). Unfortunately, a preflare spectrum was only available to test this for AT2019brs (Figure 6).

Many properties of the hosts do not align with what we expect from AGNs. The WISE colors, for example, span a broad range of $0.06-0.98 \mathrm{mag}$ (Figure 10). The IR flare associated with AT2019avd could be interpreted as a dust echo, similar to those seen in a number of TDEs (van Velzen et al. 2016). A host-subtracted SED fit to the Swift photometry of AT2019avd gives a blackbody temperature consistent with that of known TDEs, $10^{4.25} \mathrm{~K}$.

The X-ray variability of TDEs can vary erratically during a flare (e.g., Wevers et al. 2019; van Velzen et al. 2021). Although soft $\mathrm{X}$-ray excesses with $\Gamma \sim 3$ are characteristic of NLSy1s, AT2019avd displays an X-ray power-law index much higher than typically seen in NLSy1s and more characteristic of the extremely soft X-ray spectra observed in TDEs.

Based on the combination of properties shown in Figure 10, we conclude that two of the flares, AT2020hle and AT2019fdr, are better explained as TDEs in NLSy1s than AGN flares, although the interpretation is not clear cut. However, if we assume that their spectra are a combination of the host NLSy1 galaxy and the transient line emission from the TDE, then given their spectral classes here of NLSy1Fe II and NLSy1-He II, respectively, the TDE spectra themselves, in these NLSy1 galaxies, would have to be of the TDE subclasses of TDE-H (H only lines) and TDE-He (He II only lines), respectively, in order to match the observed spectra.

\subsubsection{The Extreme AGN Variability Scenario}

Extreme AGN variability, defined empirically as an optical magnitude difference of greater than $1 \mathrm{mag}$, has been seen in various optically selected samples of quasars, e.g., Lawrence (2016), Rumbaugh et al. (2018), and MacLeod et al. (2019). Graham et al. (2017) presented a sample of quasars displaying extreme variability in CRTS. Some had similar profiles and amplitudes (rising by 2-2.5 mag) but longer timescales (500-1000 days) compared to the flares presented here. For example, J002748-055559 rose by nearly $\sim 2$ mag compared to the steady level it maintained for several years prior.

The optical spectra of the transients in the sample presented here belonging to the "NLSy1-He II+N III" spectroscopic class are consistent with the properties of the observational class of flares with Bowen fluorescence established in Trakhtenbrot et al. (2019a). However, all of the transients presented here have faster fading timescales than AT2017bgt, as well as UV flares a factor of 10 less bright relative to the host levels. Trakhtenbrot et al. (2019b) stated that the fade timescale of AT2017bgt was longer than expected for a TDE. However, we note that at least one TDE in the van Velzen et al. (2021) sample (which also displayed Bowen fluorescence features) was observed to fade over nearly 15 months. 


\subsubsection{The Gravitational Microlensing Scenario}

Flares due to microlensing are expected to be observable in difference imaging surveys with the combined baseline of iPTF and ZTF. The rise portions of the light-curve shapes of all the transients measured in Section 3.1 .1 being well fit by quadratics is consistent with a lensing event; however, all but AT2020hle have a longer decay with respect to the initial rise. Microlensing by multiple foreground sources can give rise to a symmetric (with respect to the fade) double peak with a dip in the middle of the optical light curve such as that seen in AT2019pev (Hawkins 1998, 2004; Schmidt \& Wambsganss 2010). The cuspy shape of the first peak is also characteristic of microlensing light curves. AT2019avd also showed a second peak in its light curve, but the first peak was a lot more rapid and luminous than the second. To test this scenario in AT2020hle would require continuing to observe for an additional flare.

The microlensing scenario, however, would not account for the strong transient Bowen fluorescence features that appear only at late times in AT2019avd and only at early times in AT2019pev (Figure 12). Meusinger et al. (2010) explained a similar event to a background quasar with a UV flare in J004457+4123, also known as Sharov 21, being microlensed by a foreground star in M31.

Microlensing is characteristically achromatic, and therefore would be ruled out by the clear evidence for the $g-r$ color change observed in AT2019fdr.

\subsubsection{The SMBH Binary Scenario}

Variability on the timescales of years due to a binary SMBHB system would require a subparsec separation (e.g., Graham et al. 2015). In such a system, two SMBHs induce tidal torques carving out a cavity in the circumbinary accretion disk and may be surrounded by their own mini disks at sufficient separations. The interaction of accretion streams with the cavity could cause an outburst on the approximate timescales seen in this sample, which is dependent on the properties of the system. This phenomenon is seen in simulations of SMBH binaries (e.g., Ryan \& MacFadyen 2017; Gold 2019).

We see evidence of offset narrow Balmer emission lines in the spectra of AT2019fdr and AT2019avd, which may indicate a significant separate physical component, although it is unclear what is contributing to those blueshifted velocities.

\section{Conclusions}

We report five nuclear flaring events associated with NLSy1s, all serendipitously ${ }^{33}$ discovered in ZTF. We measured their photometric characteristics (such as light-curve shape, $g-r$ color, and rise to peak luminosity) and spectroscopic properties. We find tentative evidence for a link between the rise time and absolute magnitude, though understanding whether this indicates a scaling relationship with $\mathrm{BH}$ mass or the timescale of radiative diffusion through the accreting material will require a larger systematic sample. We then established groupings of the objects in the sample based on analyses of the months-long follow-up campaigns of these objects. Based on observed groupings of the sample, we

\footnotetext{
33 As the Trakhtenbrot et al. (2019a) observational class was established midway through the ZTF survey, we had not been systematically filtering such events when the population became apparent in the nuclear transients alert stream search.
}

propose the following naming scheme of spectroscopic classes of such transients for use in future optical surveys: "NLSy1He II," "NLSy1-He II+N III," and "NLSy1-Fe II." We ruled out the possibility that these are Type IIn SNe occurring in NLSy1 systems. Despite the heterogeneity of the sample's properties, two of the flares presented in this work have multiwavelength characteristics that could be consistent with TDEs in NLSy1s (AT2019fdr and AT2020hle), with spectral classes of NLSy1Fe II and NLSy1-He II, respectively. This is a high TDE rate relative to quiescent galaxies, which are more abundant than NLSy1s. The prevalence of TDE candidates in the NLSy1 AGN class could be a natural result of their hosting smaller BHs compared to typical broad-line AGNs, therefore satisfying the Hills mass criterion for an observable TDE. However, without pre-event spectra and X-ray imaging to isolate the contribution of the putative TDE to the composite NLSy1 +TDE emission, flaring due to extreme AGN variability cannot be definitively ruled out. For two in the sample (AT2019pev and AT2019avd), we can rule out the simple TDE scenario from rebrightening in their light curves, and we determine that they, along with AT2019brs (which had a preflare NLSy1 spectral classification and a BH mass estimate too large to host a canonical TDE), are likely outbursts related to enhanced accretion in excess of typical AGN variability and with spectral features we classify as "NLSy1-He II+N III," and members of the Trakhtenbrot et al. (2019a) class of AGN flares.

Given this sample, together with the growing number of interesting rapid optical transients associated with NLSy1s we reviewed in the literature, we posed the question of why such environments are observed to preferentially host these outbursts. Given the relative fraction of NLSy1s found with respect to other AGN classes in spectroscopic surveys such as SDSS ( 15\%; e.g., Zhou et al. 2006; Rakshit et al. 2017), there is likely an underlying factor enhancing this rate. We suggest four different possible explanations for this enhancement:

1. A selection bias due to shorter timescales for lower-mass BH systems (like NLSy1s), which are therefore more likely to be captured using transient-detection methods that systematically ignore slower events within the baseline of wide-field optical surveys,

2. A systematic disregard of smooth flares in broad-line AGNs during transient searches, or

3. A true intrinsic rate enhancement due to instabilities causing rapid changes in the observable environments or accretion efficiencies of these systems.

Follow-up strategies of optical transients in AGNs that are similarly ambiguous at early times may stand to benefit from the framework we offer here. We hope this classification scheme will guide real-time predictions for the potential future behavior of large-amplitude flares in NLSy1s, which are clearly an interesting population for future study. The next step will be to perform a systematic study of the variability of NLSy1s detected in ZTF to assess the completeness and rate of this sample of transients with smoothly flaring light curves and compare to a sample of broad-line AGNs. Expanding on the small number of unusual transients associated with NLSy1s not only sheds light on the parameter space in which they reside but also provides the framework for a decision tree for understanding such outbursts when they are inevitably captured 
at higher rates in upcoming wide-field surveys. This will be imperative to establish in advance of larger and deeper surveys such as ZTF Phase II and the Vera C. Rubin Observatory (formerly known as LSST; Ivezić, 2019) to which the timescales of these flares are well suited. Continued multiwavelength monitoring of the entire sample will be important to determine the host properties for those with sparse data prior to the transient and for understanding the evolution and nature of these flares.

We acknowledge and thank our reviewer for thorough and helpful feedback, which greatly enhanced the clarity of the presentation of this work. We would like to thank S. Mattila, L. Foschini, E.F. Borra, and A. Dittmann for useful comments. S.G. is supported in part by NSF CAREER grant 1454816. The work of D.S. was carried out at the Jet Propulsion Laboratory, California Institute of Technology, under a contract with NASA. We thank C. Barbarino for reducing the Nordic Optical Telescope observation of AT2019fdr. We thank R. Foley for contributing to the Lick Shane KAST observation of AT2019pev.

Based on observations obtained with the Samuel Oschin Telescope 48 inch and the 60 inch Telescope at the Palomar Observatory as part of the Zwicky Transient Facility project. ZTF is supported by the National Science Foundation under grant No. AST-1440341 and a collaboration including Caltech, IPAC, the Weizmann Institute for Science, the Oskar Klein Center at Stockholm University, the University of Maryland, the University of Washington, Deutsches Elektronen-Synchrotron and Humboldt University, Los Alamos National Laboratories, the TANGO Consortium of Taiwan, the University of Wisconsin at Milwaukee, and Lawrence Berkeley National Laboratories. Operations are conducted by COO, IPAC, and UW. This work was supported by the GROWTH project funded by the National Science Foundation under grant No. 1545949. The SED Machine is based upon work supported by the National Science Foundation under grant No. 1106171. The ZTF forced photometry service was funded under the HeisingSimons Foundation grant No. 12540303 (PI: Graham).
These results made use of the Discovery Channel Telescope at Lowell Observatory. Lowell is a private, nonprofit institution dedicated to astrophysical research and public appreciation of astronomy and operates the DCT in partnership with Boston University, the University of Maryland, the University of Toledo, Northern Arizona University, and Yale University. The upgrade of the DeVeny optical spectrograph has been funded by a generous grant from John and Ginger Giovale. Based on observations made with the Nordic Optical Telescope, owned in collaboration by the University of Turku and Aarhus University, and operated jointly by Aarhus University, the University of Turku, and the University of Oslo, representing Denmark, Finland, and Norway, the University of Iceland and Stockholm University at the Observatorio del Roque de los Muchachos, La Palma, Spain, of the Instituto de Astrofisica de Canarias.

This research has made use of data obtained through the High Energy Astrophysics Science Archive Research Center Online Service, provided by the NASA/Goddard Space Flight Center. We acknowledge the use of public data from the Swift data archive.

This publication makes use of data products from the Widefield Infrared Survey Explorer, which is a joint project of the University of California, Los Angeles, and the Jet Propulsion Laboratory/California Institute of Technology, funded by the National Aeronautics and Space Administration.

Facilities: PO:1.2m, PO:1.5m, Hale, Swift(XRT and UVOT), DCT, NOT, Shane, Liverpool:2m.

Software: Pyraf (Science Software Branch at STScI 2012), Lmfit (Newville et al. 2016), HEAsoft (NASA High Energy Astrophysics Science Archive Research Center (Heasarc) 2014), PIMMS (Mukai 1993).

\section{Appendix}

The light curves in Figure 11 are from the second IPAC data release of ZTF forced photometry. Figure 12 shows the region of interest around $\mathrm{HII}, \mathrm{H} \beta+[\mathrm{O}$ III] , and the Fe II complex for all follow-up spectra taken of the sample. 


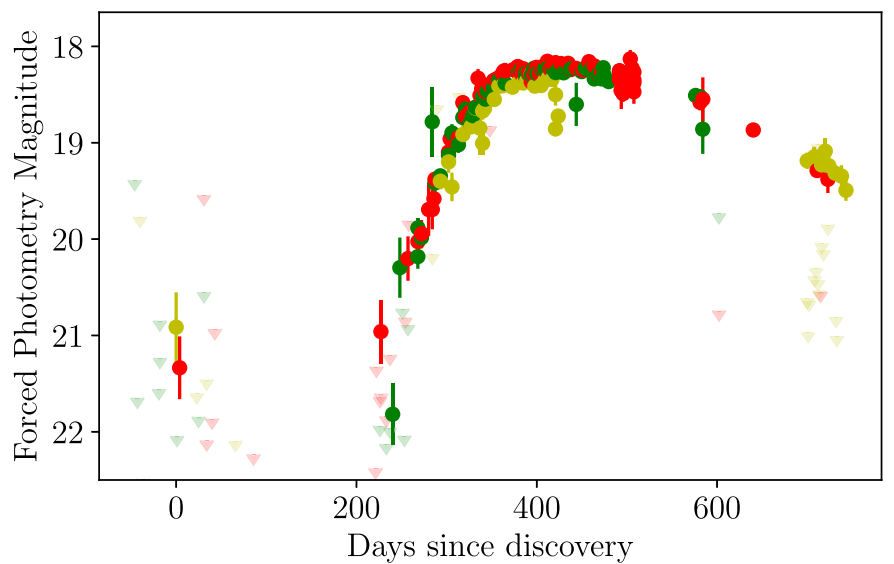

(a) AT2019brs

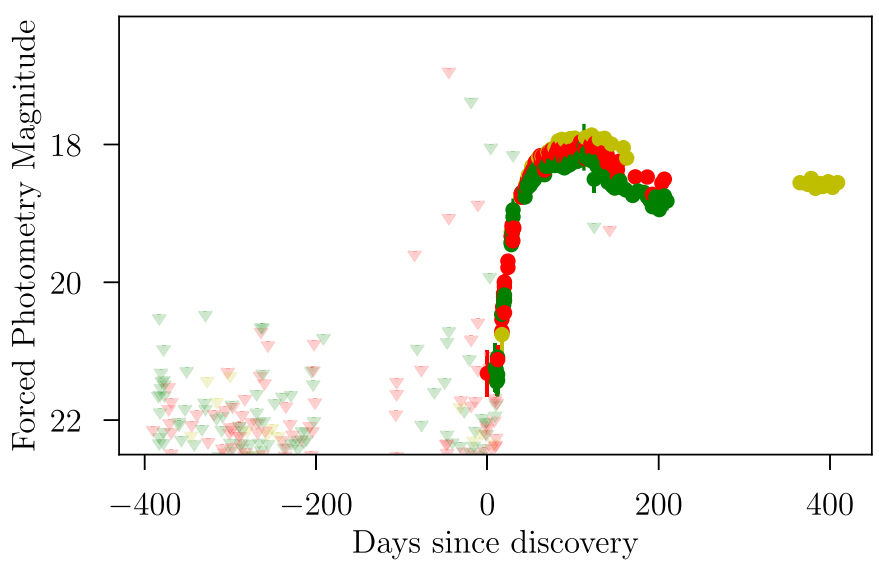

(c) AT2019fdr

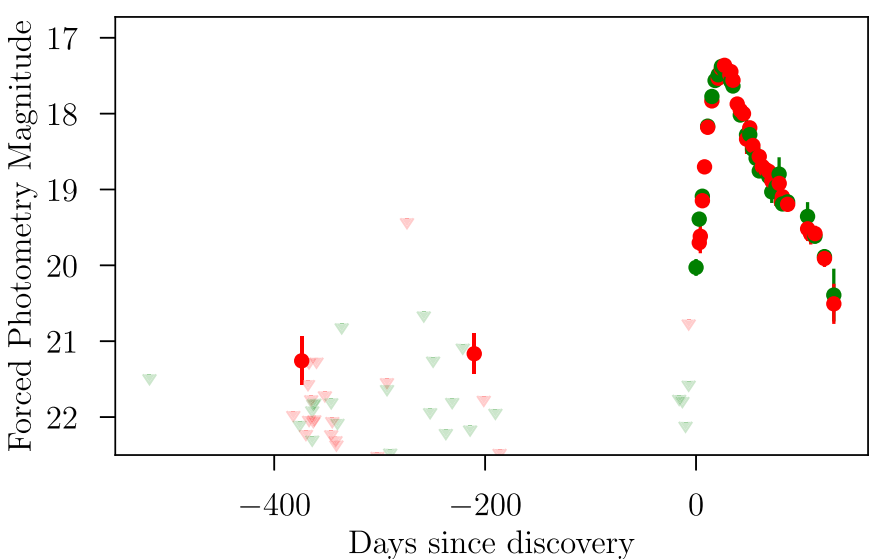

(b) AT2019pev

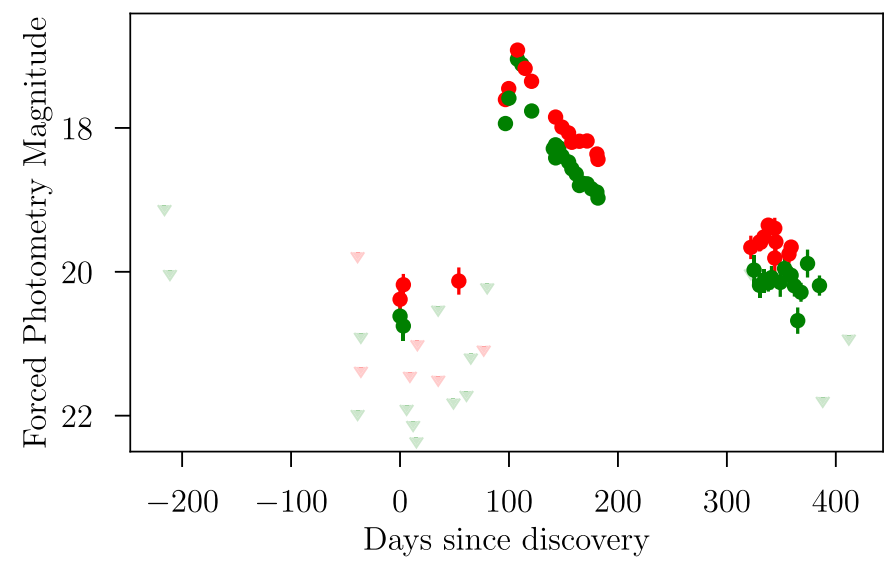

(d) AT2019avd

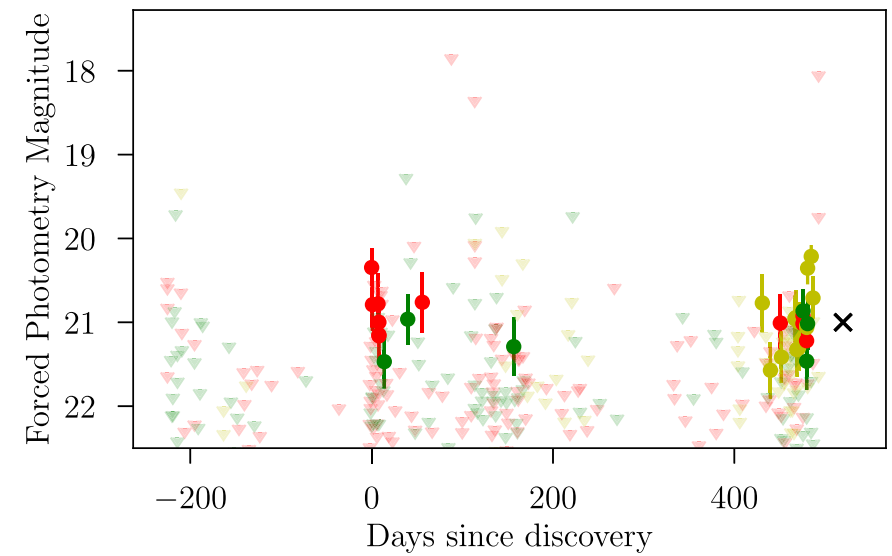

(e) AT2020hle

Figure 11. Forced photometry of the sample from ZTF Data Release 3. Colors correspond to $r$-, $g$-, and $i$-band $3 \sigma$ detections, and triangles correspond to $5 \sigma$ upper limits. An " $\times$ " marks the rise to peak in the difference imaging light curve of AT2020hle, which was discovered in data too recent to be included in the ZTF DR3, and therefore only shows the flux level of the host galaxy. The data points in the light curves beyond 2020 will be released in the final ZTF photometry data release. 


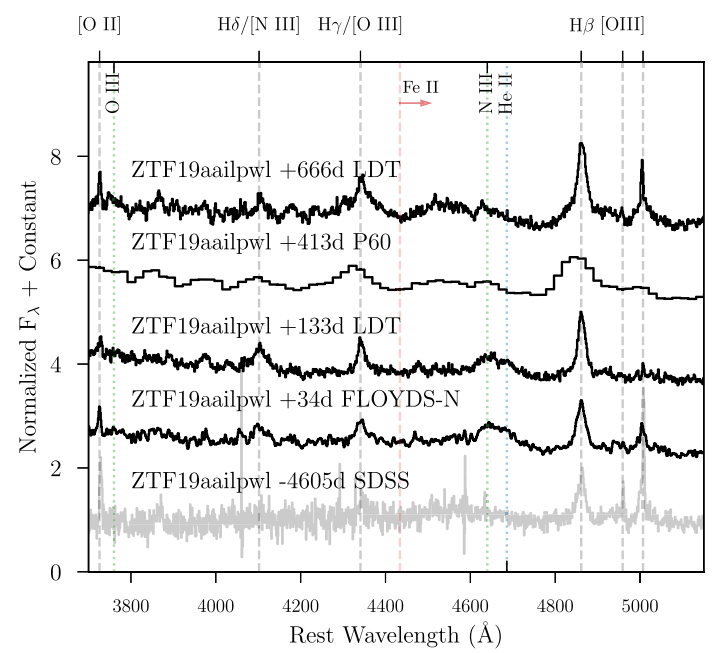

(a) AT2019brs

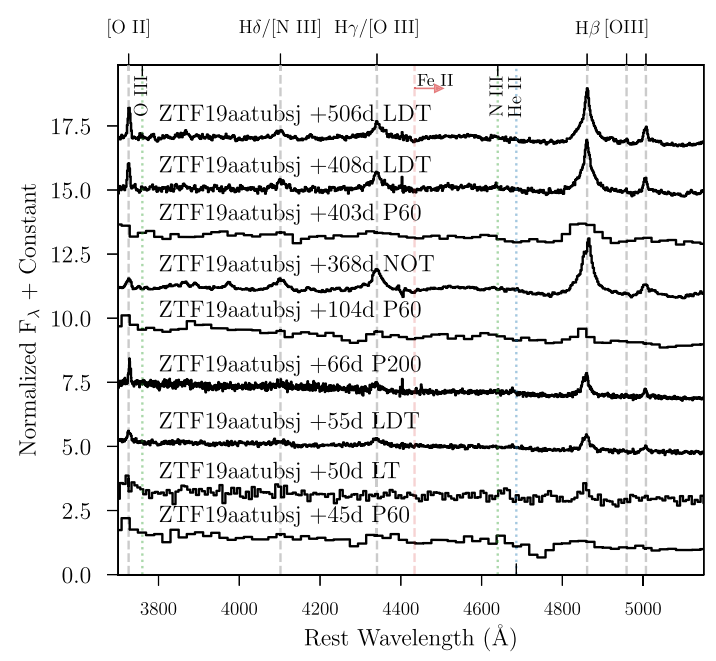

(c) AT2019fdr

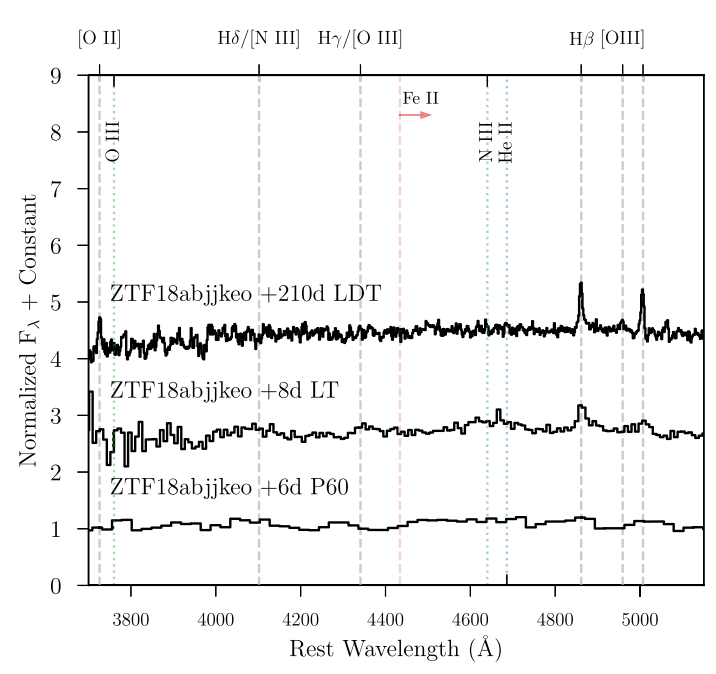

(e) AT2020hle

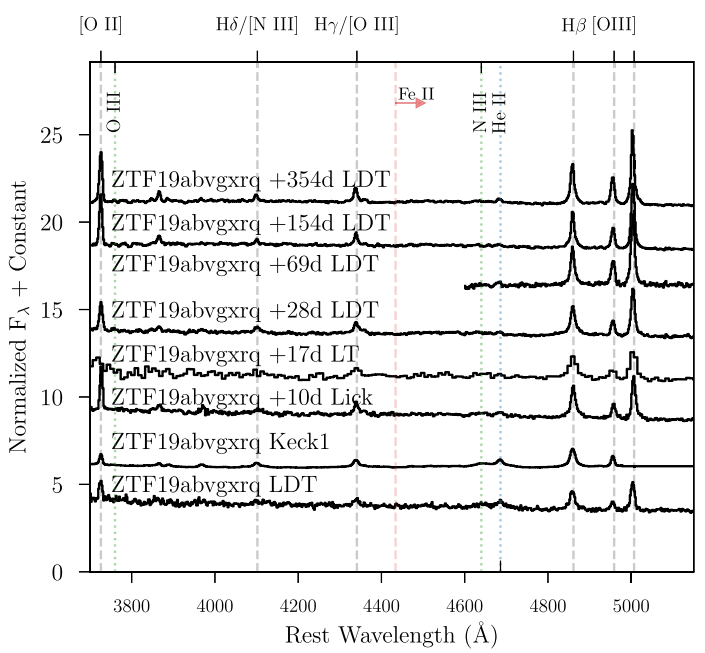

(b) AT2019pev

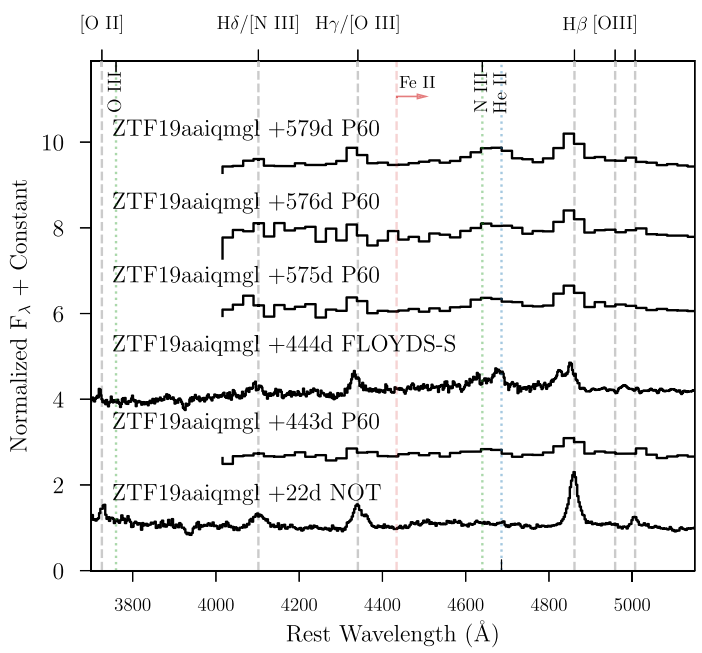

(d) AT2019avd

Figure 12. Spectroscopic follow-up of the sample summarized in Table 1, showing the evolution of the H II, H $\beta$, and Fe II line complexes. 


\section{ORCID iDs}

Sara Frederick (10) https://orcid.org/0000-0001-9676-730X Suvi Gezari (1) https://orcid.org/0000-0003-3703-5154 Matthew J. Graham (으 https://orcid.org/0000-0002-3168-0139 Jesper Sollerman (ib https://orcid.org/0000-0003-1546-6615 Sjoert van Velzen (10 https://orcid.org/0000-0002-3859-8074 Daniel A. Perley (i) https://orcid.org/0000-0001-8472-1996 Daniel Stern iㅏ https://orcid.org/0000-0003-2686-9241 Charlotte Ward (1) https://orcid.org/0000-0002-4557-6682 Erica Hammerstein (1) https://orcid.org/0000-0002-5698-8703 Tiara Hung (i) https://orcid.org/0000-0002-9878-7889 Lin Yan (1) https://orcid.org/0000-0003-1710-9339 Igor Andreoni iㅏ https://orcid.org/0000-0002-8977-1498 Eric C. Bellm (i) https://orcid.org/0000-0001-8018-5348 Dmitry A. Duev (1) https://orcid.org/0000-0001-5060-8733 Marek Kowalski @i) https://orcid.org/0000-0001-8594-8666 Ashish A. Mahabal (i) https://orcid.org/0000-0003-2242-0244 Frank J. Masci $\odot$ https://orcid.org/0000-0002-8532-9395 Michael Medford (1) https://orcid.org/0000-0002-7226-0659 Ben Rusholme (i) https://orcid.org/0000-0001-7648-4142 Roger Smith (1) https://orcid.org/0000-0001-7062-9726

\section{References}

Abolfathi, B., Aguado, D. S., Aguilar, G., et al. 2018, ApJS, 235, 42 Ai, Y. L., Yuan, W., Zhou, H. Y., et al. 2010, ApJL, 716, L31 Arcavi, I., Trakhtenbrot, B., \& Hiramatsu, D. 2019, TNSAN, 4, 1 Arnaud, K. A. 1996, in ASP Conf. Ser. 101, Astronomical Data Analysis Software and Systems V, ed. G. H. Jacoby \& J. Barnes (San Francisco, CA: ASP), 17

Assef, R. J., Stern, D., Kochanek, C. S., et al. 2013, ApJ, 772, 26

Baldi, R. D., Capetti, A., Robinson, A., Laor, A., \& Behar, E. 2016, MNRAS, 458, L69

Barth, A. J., Pancoast, A., Bennert, V. N., et al. 2013, ApJ, 769, 128

Bellm, E. C., Kulkarni, S. R., Barlow, T., et al. 2019b, PASP, 131, 068003

Bellm, E. C., Kulkarni, S. R., Graham, M. J., et al. 2019a, PASP, 131, 018002 Bellm, E. C., \& Sesar, B. 2016, pyraf-dbsp: Reduction pipeline for the Palomar Double Beam Spectrograph ascl, 1602, 002, ascl:1602.002

Bianchi, L., Shiao, B., \& Thilker, D. 2017, ApJS, 230, 24

Blagorodnova, N., Cenko, S. B., Kulkarni, S. R., et al. 2019, ApJ, 873, 92 Blagorodnova, N., Neill, J. D., Walters, R., et al. 2018, PASP, 130, 035003 Blanchard, P. K., Nicholl, M., Berger, E., et al. 2017, ApJ, 843, 106 Boller, T., Brandt, W. N., \& Fink, H. 1996, A\&A, 305, 53

Bruch, R. J., Gal-Yam, A., Schulze, S., et al. 2021, ApJ, 912, 46

Chambers, K. C., Magnier, E. A., Metcalfe, N., et al. 2016, arXiv: 1612.05560 Chan, C.-H., Piran, T., \& Krolik, J. H. 2020, arXiv:2004.06234

Chan, C.-H., Piran, T., Krolik, J. H., \& Saban, D. 2019, ApJ, 881, 113

Chen, P. S., Liu, J. Y., \& Shan, H. G. 2017, NewA, 54, 30

Decarli, R., Labita, M., Treves, A., \& Falomo, R. 2008, MNRAS, 387, 1237

Drake, A. J., Djorgovski, S. G., Mahabal, A., et al. 2009, ApJ, 696, 870

Drake, A. J., Djorgovski, S. G., Mahabal, A., et al. 2011, ApJ, 735, 106

Evans, P. A., Beardmore, A. P., Page, K. L., et al. 2009, MNRAS, 397, 1177 Forster, K., \& Halpern, J. P. 1996, ApJ, 468, 565

Frederick, S., Gezari, S., Graham, M. J., et al. 2019, ApJ, 883, 31

Frederick, S., Kara, E., Reynolds, C., Pinto, C., \& Fabian, A. 2018, ApJ, 867, 67

Gallegos-Garcia, M., Law-Smith, J., \& Ramirez-Ruiz, E. 2018, ApJ, 857, 109 Gallo, L. C. 2006, MNRAS, 368, 479

Gehrels, N., Chincarini, G., Giommi, P., et al. 2004, ApJ, 611, 1005 Gezari, S., van Velzen, S., Perley, D., et al. 2019, ATel, 13127, 1

Gold, R. 2019, Galax, 7, 63

Graham, M. J., Djorgovski, S. G., Drake, A. J., et al. 2017, MNRAS, 470, 4112 Graham, M. J., Djorgovski, S. G., Stern, D., et al. 2015, MNRAS, 453, 1562 Graham, M. J., Kulkarni, S. R., Bellm, E. C., et al. 2019, PASP, 131, 078001 Graham, M. J., Ross, N. P., Stern, D., et al. 2020, MNRAS, 491, 4925 Gromadzki, M., Hamanowicz, A., Wyrzykowski, L., et al. 2019, A\&A, 622, L2

Grupe, D., Komossa, S., Leighly, K. M., \& Page, K. L. 2010, ApJS, 187, 64 Guainazzi, M., Nicastro, F., Fiore, F., et al. 1998, MNRAS, 301, L1 Halpern, J. P., \& Eracleous, M. 1994, ApJL, 433, L17
Hammerstein, E., Gezari, S., van Velzen, S., et al. 2021, ApJL, 908, L20

Hawkins, M. R. S. 1998, A\&A, 340, L23

Hawkins, M. R. S. 2004, BaltA, 13, 642

Hills, J. G. 1975, Natur, 254, 295

Ivezić, Ž., Kahn, S. M., Tyson, J. A., et al. 2019, ApJ, 873, 111 Jayasinghe, T., Stanek, K. Z., Kochanek, C. S., et al. 2019, MNRAS, 485, 961 Jiang, N., Wang, T., Mou, G., et al. 2019, ApJ, 871, 15

Kankare, E., Kotak, R., Mattila, S., et al. 2017, NatAs, 1, 865

Kara, E., Pasham, D., Gendreau, K., \& Arzoumanian, Z. 2019, ATel, 13132, 1 Kasliwal, M. M., Cannella, C., Bagdasaryan, A., et al. 2019, PASP, 131, 038003

Kaspi, S., Smith, P. S., Netzer, H., et al. 2000, ApJ, 533, 631

Khazov, D., Yaron, O., Gal-Yam, A., et al. 2016, ApJ, 818, 3

Kiewe, M., Gal-Yam, A., Arcavi, I., et al. 2012, ApJ, 744, 10

Klimek, E. S., Gaskell, C. M., \& Hedrick, C. H. 2004, ApJ, 609, 69

Kochanek, C. S. 2016, MNRAS, 458, 127

LaMassa, S. M., Cales, S., Moran, E. C., et al. 2015, ApJ, 800, 144 Lawrence, A. 2016, arXiv:1605.09331v1

Lawrence, A., Gezari, S., Elvis, M., et al. 2012, in Tidal Disruption Events and AGN Outbursts, EPJ Web of Conferences, vol. 39, ed. R. Saxton \& S. Komossa, 03002

Liu, H.-Y., Yuan, W., Dong, X.-B., Zhou, H., \& Liu, W.-J. 2018a, ApJS, 235,40

Liu, X., Dittmann, A., Shen, Y., \& Jiang, L. 2018b, ApJ, 859, 8

Liu, Z., Li, D., Liu, H.-Y., et al. 2020, ApJ, 894, 93

Lunnan, R., Yan, L., Perley, D. A., et al. 2020, ApJ, 901, 61

MacLeod, C. L., Green, P. J., Anderson, S. F., et al. 2019, ApJ, 874, 8

MacLeod, C. L., Ross, N. P., Lawrence, A., et al. 2016, MNRAS, 457, 389

Malyali, A., Rau, A., Arcodia, R., et al. 2020, ATel, 13712, 1

Malyali, A., Rau, A., Merloni, A., et al. 2021, A\&A, 647, A9

Marconi, A., Axon, D. J., Maiolino, R., et al. 2008, ApJ, 678, 693

Martin, D. C., Fanson, J., Schiminovich, D., et al. 2005, ApJL, 619, L1

Masci, F. J., Laher, R. R., Rusholme, B., et al. 2019, PASP, 131, 018003

Mathur, S., Auchettl, K., Kochanek, C. S., et al. 2019, ATel, 13213, 1

Mathur, S., Kuraszkiewicz, J., \& Czerny, B. 2001, NewA, 6, 321

McLure, R. J., \& Dunlop, J. S. 2002, MNRAS, 331, 795

Merloni, A., Dwelly, T., Salvato, M., et al. 2015, MNRAS, 452, 69

Meusinger, H., Henze, M., Birkle, K., et al. 2010, A\&A, 512, A1

Miller, H. R., Ferrara, E. C., McFarland, J. P., et al. 2000, NewAR, 44, 539

Miller, J. M., Zoghbi, A., Reynolds, M., et al. 2019, ATel, 13163, 1

Molthagen, K., Bade, N., \& Wendker, H. J. 1998, A\&A, 331, 925

Moriya, T. J., Sorokina, E. I., \& Chevalier, R. A. 2018, SSRv, 214, 59

Mukai, K. 1993, Legacy, 3, 21

NASA High Energy Astrophysics Science Archive Research Center (Heasarc). 2014, HEAsoft: Unified Release of FTOOLS and XANADU. ascl:1408.004

Neustadt, J. M. M., Holoien, T. W. S., Kochanek, C. S., et al. 2020, MNRAS, 494, 2538

Newville, M., Stensitzki, T., Allen, D. B., et al. 2016, Lmfit: Non-Linear LeastSquare Minimization and Curve-Fitting for Python, ascl:1606.014

Nikołajuk, M., Czerny, B., \& Gurynowicz, P. 2009, MNRAS, 394, 2141

Nordin, J., Brinnel, V., van Santen, J., et al. 2019, A\&A, 631, A147

Pasham, D., Gendreau, K., Arzoumanian, Z., et al. 2020, ATel, 14036, 1

Pasham, D. R., Wang, Y., Remillard, R., et al. 2021, ATel, 14664, 1

Patterson, M. T., Bellm, E. C., Rusholme, B., et al. 2019, PASP, 131, 018001

Payne, A. V., Shappee, B. J., Hinkle, J. T., et al. 2021, ApJ, 910, 125

Perley, D. A. 2019, PASP, 131, 084503

Peterson, B. M., \& Ferland, G. J. 1986, Natur, 324, 345

Pogge, R. W. 2000, NewAR, 44, 381

Pounds, K. A., Done, C., \& Osborne, J. P. 1995, MNRAS, 277, L5

Predehl, P., Andritschke, R., Arefiev, V., et al. 2021, A\&A, 647, A1

Rafter, S. E., Kaspi, S., Chelouche, D., et al. 2013, ApJ, 773, 24

Rakshit, S., Stalin, C. S., Chand, H., \& Zhang, X.-G. 2017, ApJS, 229, 39

Rigault, M., Neill, J. D., Blagorodnova, N., et al. 2019, A\&A, 627, A115

Ross, N. P., Ford, K. E. S., Graham, M., et al. 2018, MNRAS, 480, 4468

Ruan, J. J., Anderson, S. F., Cales, S. L., et al. 2016, ApJ, 826, 188

Rumbaugh, N., Shen, Y., Morganson, E., et al. 2018, ApJ, 854, 160

Runnoe, J. C., Brotherton, M. S., \& Shang, Z. 2012, MNRAS, 422, 478

Runnoe, J. C., Cales, S., Ruan, J. J., et al. 2016, MNRAS, 455, 1691

Ryan, G., \& MacFadyen, A. 2017, ApJ, 835, 199

Saxton, C. J., Perets, H. B., \& Baskin, A. 2018, MNRAS, 474, 3307

Saxton, R. D., Read, A. M., Esquej, P., et al. 2008, A\&A, 480, 611

Schmidt, R. W., \& Wambsganss, J. 2010, GReGr, 42, 2127

Science Software Branch at STScI 2012, PyRAF: Python alternative for IRAF, ascl: 1207.011

Shen, Y., Richards, G. T., Strauss, M. A., et al. 2011, ApJS, 194, 45 
Smartt, S. J., Smith, K. W., McBrien, O., et al. 2019, TNSAN, 33, 1 Sollerman, J., Fransson, C., Barbarino, C., et al. 2020, A\&A, 643, A79

Sollerman, J., Taddia, F., Arcavi, I., et al. 2019, A\&A, 621, A30

Soumagnac, M. T., \& Ofek, E. O. 2018, PASP, 130, 075002

Stein, R., van Velzen, S., Kowalski, M., et al. 2021, NatAs, 5, 510

Stern, D., Assef, R. J., Benford, D. J., et al. 2012, ApJ, 753, 30

Stern, D., McKernan, B., Graham, M. J., et al. 2018, ApJ, 864, 27

Tadhunter, C., Spence, R., Rose, M., Mullaney, J., \& Crowther, P. 2017, NatAs, 1, 0061

Tananbaum, H., Avni, Y., Branduardi, G., et al. 1979, ApJL, 234, L9

Terlevich, R., Tenorio-Tagle, G., Franco, J., \& Melnick, J. 1992, MNRAS, 255,713

Trakhtenbrot, B., Arcavi, I., MacLeod, C. L., et al. 2019b, ApJ, 883, 94

Trakhtenbrot, B., Arcavi, I., Ricci, C., et al. 2019a, NatAs, 3, 242

Trakhtenbrot, B., Arcavi, I., Ricci, C., \& Burke, J. 2020, TNSAN, 105, 1
Uttley, P., McHardy, I. M., Papadakis, I. E., Guainazzi, M., \& Fruscione, A. 1999, MNRAS, 307, L6

van Velzen, S., Gezari, S., Cenko, S. B., et al. 2019, ApJ, 872, 198

van Velzen, S., Gezari, S., Hammerstein, E., et al. 2021, ApJ, 908, 4

van Velzen, S., Holoien, T. W. S., Onori, F., Hung, T., \& Arcavi, I. 2020, SSRv, 216, 124

van Velzen, S., Mendez, A. J., Krolik, J. H., \& Gorjian, V. 2016, ApJ, 829, 19 Voges, W., Aschenbach, B., Boller, T., et al. 1999, A\&A, 349, 389

Wang, T., Brinkmann, W., \& Bergeron, J. 1996, A\&A, 309, 81

Wevers, T., Pasham, D. R., van Velzen, S., et al. 2019, MNRAS, 488, 4816

Xu, D., Komossa, S., Zhou, H., et al. 2012, AJ, 143, 83

Yan, L., Perley, D., Schulze, S., et al. 2020, ApJL, 902, L8

Yan, L., Wang, T., Jiang, N., et al. 2019, ApJ, 874, 44

Yaron, O., \& Gal-Yam, A. 2012, PASP, 124, 668

Zhou, H., Wang, T., Yuan, W., et al. 2006, ApJS, 166, 128 